\title{
Broad Ultrastructural and Transcriptomic Changes Underlie the Multinucleated Giant Hemocyte Mediated Innate Immune Response against Parasitoids
}

\author{
Gyöngyi Cinege ${ }^{a}$ Lilla B. Magyara, b Attila L. Kovács ${ }^{c} \quad$ Zita Lerner $^{a}$ b \\ Gábor Juhász ${ }^{c}$ David Lukacsovich ${ }^{d}$ Jochen Winterer ${ }^{d}$ Tamás Lukacsovich $^{d}$ \\ Zoltán Hegedűs ${ }^{e, f}$ Éva Kurucz ${ }^{a} \quad$ Dan Hultmark $^{g}$ Csaba Földy ${ }^{d}$ István Andóa \\ anstitute of Genetics, Innate Immunity Group, Immunology Unit, Biological Research Centre, Szeged, Hungary; \\ ${ }^{b}$ Doctoral School of Biology, University of Szeged, Szeged, Hungary; 'Department of Anatomy, Cell and

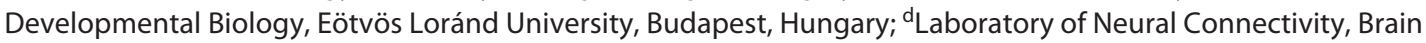 \\ Research Institute, University of Zurich, Zurich, Switzerland; 'Laboratory of Bioinformatics, Biological Research \\ Centre, Szeged, Hungary; fDepartment of Biochemistry and Medical Chemistry, Medical School, University of Pécs, \\ Pécs, Hungary; ${ }^{9}$ Department of Molecular Biology, Umea University, Umea, Sweden
}

\section{Keywords}

Multinucleated giant hemocyte - Encapsulation •

Drosophila $\cdot$ Transcriptome $\cdot$ Innate immunity

\begin{abstract}
Multinucleated giant hemocytes (MGHs) represent a novel type of blood cell in insects that participate in a highly efficient immune response against parasitoid wasps involving isolation and killing of the parasite. Previously, we showed that circulating MGHs have high motility and the interaction with the parasitoid rapidly triggers encapsulation. However, structural and molecular mechanisms behind these processes remained elusive. Here, we used detailed ultrastructural analysis and live cell imaging of MGHs to study encapsulation in Drosophila ananassae after parasitoid wasp infection. We found dynamic structural changes, mainly driven by the formation of diverse vesicular systems and newly developed complex intracytoplasmic membrane structures, and abun-
\end{abstract}

karger@karger.com www.karger.com/jin

Karger!

GOPEN ACCESS
(C) 2021 The Author(s)

Published by S. Karger AG, Basel

This is an Open Access article licensed under the Creative Commons Attribution-NonCommercial-4.0 International License (CC BY-NC) (http://www.karger.com/Services/OpenAccessLicense), applicable to the online version of the article only. Usage and distribution for commercial purposes requires written permission. dant generation of giant cell exosomes in MGHs. In addition, we used RNA sequencing to study the transcriptomic profile of MGHs and activated plasmatocytes $72 \mathrm{~h}$ after infection, as well as the uninduced blood cells. This revealed that differentiation of MGHs was accompanied by broad changes in gene expression. Consistent with the observed structural changes, transcripts related to vesicular function, cytoskeletal organization, and adhesion were enriched in MGHs. In addition, several orphan genes encoding for hemolysin-like proteins, pore-forming toxins of prokaryotic origin, were expressed at high level, which may be important for parasitoid elimination. Our results reveal coordinated molecular and structural changes in the course of MGH differentiation and parasitoid encapsulation, providing a mechanistic model for a powerful innate immune response.

(C) 2021 The Author(s)

Published by S. Karger AG, Basel

Gyöngyi Cinege and Lilla B. Magyar contributed equally to this work.
Correspondence to:

Csaba Földy, foldy@ hifo.uzh.ch

István Andó, ando.istvan @brc.hu 


\section{Introduction}

Drosophila melanogaster is frequently used to study fundamental immune responses and host-pathogen interactions because innate immune mechanisms are highly similar in insects and vertebrates [1-3]. This species possesses an open circulatory system with 2 types of blood cells: spherical plasmatocytes and crystal cells, which represent $95 \%$ and $5 \%$ of the circulating hemocyte pool, respectively. After attack by parasitoid wasps or wounding, large, flattened cells differentiate into a third cell type, called lamellocytes. These 3 blood cell types execute complementary functions in immune protection [4-8]. Plasmatocytes play a role in the phagocytosis of microorganisms and produce antimicrobial peptides and matrix proteins [9], whereas lamellocytes form capsules around the eggs or larvae of parasitoid wasps. Crystal cells and lamellocytes produce and carry prophenoloxidases, which after injury or infection are transformed into active phenoloxidases, responsible for a melanization reaction at wound sites and around parasitoids $[10,11]$.

Previously, we found that in species of the ananassae subgroup and in Zaprionus indianus belonging to the vittiger subgroup of Drosophilidae, the immune response is highly effective and remarkably different from that in $D$. melanogaster $[12,13]$. In these species, after infection by parasitoids, giant cells possessing several nuclei, termed as multinucleated giant hemocytes (MGHs), differentiate and encapsulate the invader [12]. Multinucleation has been observed in various cells of living organisms, and their appearance is frequently associated with infections $[14,15]$. Known examples included the Langhans multinucleated giant cells that differentiate around foreign bodies during granulomatous inflammation [16, 17], multinucleated syncytia formed by SARS-CoV-2 coronavirus-infected cells $[18,19]$, multinucleated hemocytes in Mercenaria (Mollusc) species after pollutant exposure [20], multinucleated cells in the roots of plants after nematode infection [21], and multinucleated epidermal cells around the wound of Drosophila larvae [22], which help seal off the wound site by eliminating intercellular spaces. Multinucleation, as a form of genome amplification, significantly increases the genetic material within 1 cytoplasm, which provides an elevated level of protein expression and intensified metabolism for these cells.

Similar to lamellocytes, MGHs encapsulate parasitoid wasp eggs and larvae, but do not participate in bacterial phagocytosis, the process of which is mediated by plasmatocytes $[12,13]$. However, MGHs are highly motile, and their presence is associated with a remarkably effi- cient protection against parasitoid wasps [12, 13, 23, 24]. In addition, 1 key step of the immune protection is different between MGHs and lamellocytes. In D. melanogaster, the capsule formed by lamellocytes around the parasitoid wasp is melanized. Melanization generates reactive oxygen species and creates a mechanical barrier, which is important for a successful defense $[10,11]$. Melanization requires 3 prophenoloxidases: $\mathrm{PPO} 1, \mathrm{PPO} 2$, and $\mathrm{PPO} 3$. Of these, PPO2, synthesized by crystal cells, and PPO3, expressed by lamellocytes, are directly involved in capsule melanization around parasitoids [11]. By contrast, the $D$. ananassae genome has orthologs only for PPO1 (FBgn0090249, 79.7\% identity) and PPO2 (FBgn0089750, 92.3\% identity), but not for PPO3 [11]. In accordance with this, in species of the ananassae subgroup, capsules generated by MGHs are not melanized [12].

To gain insights into the particular immune mechanisms of $D$. ananassae mediated by MGHs, we used electron microscopy and gene expression analysis to unveil mechanisms behind the function of these cells. We demonstrate that during their differentiation, various characteristic morphological features develop in MGHs. Most notable are the changes in surface membranes, the development of a unique, new intracytoplasmic membrane system, generation of giant cell exosomes (GCEs), and enrichment of lipid droplets and microtubules. In line with these morphological changes, using RNAseq transcriptome analysis, we show expression changes in genes related to cell surface morphology and vesicular systems in MGHs. Specifically, we found that several enriched genes were associated with the composition, function, and transport of vesicles and lipid droplets. Moreover, genes involved in cytoskeletal organization, motility, and adhesion became enriched, consistent with the high motility and adherent features of these cells. Finally, our transcriptomic analyses revealed that neither of the melanization-related prophenoloxidases was expressed in MGHs, which underscores the notion that the MGH response in D. ananassae is fundamentally different from that of lamellocytes in D. melanogaster. Together, our data provide novel insights into the immune response mediated by MGHs, which rapidly gain cellular mass, capable of extraordinary fast movements, and effectively kill invading parasitoids.

\section{Materials and Methods}

Insect Stocks and Culturing

D. ananassae wild type (14024-0371.13) had been obtained from UC San Diego Drosophila stock center and kept at $25^{\circ} \mathrm{C}$ on standard yeast-cornmeal food. The Leptopilina boulardi wasp 
strain G486 was kindly provided by Prof. Todd Schlenke (University of Arizona, Tucson, AZ, USA). Wasps were maintained on D. melanogaster Oregon $R$.

\section{Parasitization Assay}

$D$. ananassae larvae were infected with $L$. boulardi parasitoid wasps. Sixty second-instar larvae were transferred into a vial containing standard fly food and were exposed to 15 female wasps for $6 \mathrm{~h}$. Seventy-two hours following the wasp infection, for further experiments, we selected larvae with melanized spots at the site of oviposition on the cuticle.

\section{Electron Microscopy}

Dissection of the larvae was carried out in the fixation solution for both conventional and immune electron microscopy. Further treatment for conventional samples was as follows. Fixation was carried out for $48 \mathrm{~h}$ at $4^{\circ} \mathrm{C}$ in the fixative containing: $0.5 \%$ glutaraldehyde (EM Grade, Polysciences), 4\% formaldehyde (Polysciences), $2 \mathrm{mM} \mathrm{CaCl}_{2}$, and $1 \%$ sucrose in $0.1 \mathrm{~mol} / \mathrm{L} \mathrm{Na}$ cacodylate buffer (Polysciences). Fixed samples were embedded in $4 \%$ agar, washed in $0.1 \mathrm{~mol} / \mathrm{L} \mathrm{Na}$ cacodylate 3 times for $10 \mathrm{~min}$ each, stained with $1 \% \mathrm{OsO}_{4}$ (Polysciences) in $0.1 \mathrm{~mol} / \mathrm{L} \mathrm{Na}$ cacodylate for $1 \mathrm{~h}$, rinsed with $10 \%$ acetone twice, and stained with $2 \%$ uranyl acetate (EM, TAAB Laboratory, and Microscopy) in distilled water for 2 h. Dehydration of samples in a graded series of ethanol $(50 \%, 70 \%$, $96 \%$ twice and 100\% 3 times each), and then in propylene oxide (Sigma) for $15 \mathrm{~min}$. Infiltration was carried out in propylene oxide:Durcupan ACM (Sigma) epoxy resin, 2:1 overnight, in propylene oxide:Durcupan 1:2, and in full Durcupan ACM for $2 \mathrm{~h}$ each. Polymerization was done for $48 \mathrm{~h}$ at $60^{\circ} \mathrm{C}$. For Ni-DAB electron microscopic immunolabeling, the samples were kept $1 \mathrm{~h}$ at room temperature (RT) in a fixative containing $0.5 \%$ glutaraldehyde (EM Grade, Polysciences) and 4\% formaldehyde (Polysciences) in distilled water. After this, the samples went through the following steps: rinsing once in PBS, washing once in TBS for $30 \mathrm{~min}$ at RT, incubating in $30 \%$ sucrose-PBS $1 \mathrm{~h}$ at $4^{\circ} \mathrm{C}$ and $1 \mathrm{~h}$ at RT, freezing by dipping into liquid nitrogen 3 times, washing in TBS twice for 15 min each at RT, incubating in $1 \% \mathrm{H}_{2} \mathrm{O}_{2}$-TBS for 10 min at RT, and washing again in TBS twice for 15 min each at RT; quenching followed in 50-50 $\mathrm{mM} \mathrm{NH}_{4} \mathrm{Cl}$-Glycin in TBS for $30 \mathrm{~min}$ at RT, then washing in TBS twice for $15 \mathrm{~min}$, and blocking in 10\% FBS-1\% BSA-TBS for $30 \mathrm{~min}$, both at RT. For immune reaction, samples were incubated overnight at $4^{\circ} \mathrm{C}$ with the $7 \mathrm{C} 5$, a $D$. ananassae MGH-specific mouse monoclonal antibody [12] and a negative control isotype-matched monoclonal antibody. The 7C5 antibody reacts with live cells, showing that it is specific to an immunological epitope in the plasma membrane (I.A., unpublished). The samples were washed with 1\% BSA-TBS, 4 times each for 10 min. As the secondary probe biotinylated goat anti-mouse antibody, 1:500 diluted in 1\% BSA-TBS, was used overnight at $4^{\circ} \mathrm{C}$, washing in $1 \%$ BSA-TBS, 4 times each for $15 \mathrm{~min}$ at RT, then incubation with HRP conjugated ABC reagent (avidin-biotin complex) diluted at 1:500 in 1\% BSA-TBS for $5 \mathrm{~h}$ at RT, and washing again in TBS 4 times for 10 min each at RT. Preincubation without $\mathrm{Ni}$-DAB was carried out next in dark for $30 \mathrm{~min}$ at RT (DAB peroxidase substrate kit SK-4100, Vector). Samples were treated with $\mathrm{Ni}-\mathrm{DAB} \mathrm{H}_{2} \mathrm{O}_{2}$ for a few minutes, until the development of dark precipitate; washed in TBS 4 times 10 min each at RT; left overnight in TBS at $4^{\circ} \mathrm{C}$; embedded in $4 \%$ agar; washed in $0.1 \mathrm{~mol} / \mathrm{L} \mathrm{Na}$ cacodylate for $1 \mathrm{~h}$ at RT; stained with $\mathrm{OsO}_{4}$ (Polysciences) in 0.1 $\mathrm{mol} / \mathrm{L}$ Na cacodylate for $1 \mathrm{~h}$; rinsed with $10 \%$ acetone twice; stained with $2 \%$ uranyl acetate (EM, TAAB Laboratory and Microscopy) in distilled water for $30 \mathrm{~min}$; and dehydrated in a graded series of ethanol (50\%, 70\%, 96\% for 2 times each, and 100\% 3 times) and then in propylene oxide (Sigma) for 15 min. Infiltration was carried out in propylene oxide:Durcupan ACM (Sigma) epoxy resin, 3:1 and 1:1 for 45 min each and 1:3 mix overnight next moved to full Durcupan ACM. Polymerization was carried out for $48 \mathrm{~h}$ at $60^{\circ} \mathrm{C}$. Sectioning of electron microscopy blocks for both standard and immunosamples was made by Reichert Ultracut ultramicrotome, and staining of sections was made in Reynolds lead citrate. Sections were viewed in a JEM-1011 JEOL transmission electron microscope and pictures taken with a Morada, Olympus camera, and iTEM software (Olympus).

\section{Visualization of Acidic Vesicles Using LysoTracker Dye}

Seventy-two hours following the L. boulardi infection, D. ananassae larvae were bled in Schneider's medium (Lonza) supplemented with $5 \%$ fetal bovine serum (GIBCO) and 0.01\% 1-phenyl 2-thiourea (Sigma) (CSM) on microscope slides and the blood cells were adhered to the glass slides for $1 \mathrm{~h}$. The encapsulated parasitoids were isolated similarly, and to avoid pipetting of the capsules, they were prepared under the microscope. The CSM medium was gently removed and the cells and capsules were washed once in PBS and then incubated in the dark with LysoTracker ${ }^{\mathrm{TM}}$ Red DND99 (Invitrogen, 1:1,000 dilution in PBS) for $3 \mathrm{~min}$. The samples were washed twice with PBS and covered with Fluoromount G medium and coverslip. The samples were immediately analyzed with an epifluorescence microscope (Zeiss Axioscope 2 MOT) or with an Olympus FV1000 confocal LSM microscope.

\section{Collection of Samples for Next-Generation RNA Sequencing}

Wasp-infected $D$. ananassae larvae $72 \mathrm{~h}$ following the infection were washed out from the vials in Drosophila Ringer solution. Blood cells were isolated in Schneider's medium (Lonza) supplemented with $5 \%$ fetal bovine serum (GIBCO) on microscope slides. Sample collection was performed as described previously [25]. Briefly, cytosol of a blood cell, attached for $30 \mathrm{~min}$ to a microscopic slide, was aspired via a glass pipette, containing $1.5 \mu \mathrm{L}$ RNase-free sterile Drosophila Ringer solution, mounted on a micromanipulator. For sample collection, the pipette holder was quickly removed from the micromanipulator and the collected cytosol, by applying positive pressure, was expelled to microtubes containing $1 \mu \mathrm{L}$ lysis buffer with an RNase inhibitor from the TAKARA Clontech SMART-Seq v4 Ultra Low Input RNA Kit for Sequencing (Cat. No. 634889). Tubes were briefly centrifuged and snap-frozen on dry ice. Cytosol of 5 plasmatocytes or 5 MGHs was pooled in 1 microtube, respectively. Overall, 5 pools were collected. Samples were stored at $-80^{\circ} \mathrm{C}$ until further processing according to the manufacturer's protocol. In parallel, 5 blood cell pools were generated, each from 50 age-matched uninfected larvae. The total RNA samples were generated by RNeasy micro kit from Qiagen.

\section{cDNA Library Preparation and Next-Generation RNA}

Sequencing

The same procedures were followed as previously $[25,26]$. mRNA was processed using Clontech's SMARTer Ultra Low RNA Input $\mathrm{v} 4$ kit. The resulting cDNA from MGH and activated plasmatocyte samples, originating from single cell isolates, were har- 
vested and analyzed on a Fragment Analyzer (Advanced Analytical). Library preparation was performed using an Illumina FC131-1024-Nextera XT DNA SMP Prep Kit (Cat. No. 1293799) according to the manufacturer's protocol. The samples were sequenced using a NextSeq 150 high-output kit (Cat. No. 20024907) in an Illumina NextSeq 500 System with $2 \times 75$ paired-end reads. The cDNA synthesis from naïve hematocytes was carried out by Clontech-Takara SMARTer cDNA synthesis kit. The resulting cDNA was then analyzed on a Fragment Analyzer (Advanced Analytical), and concentration-adjusted samples were further processed by a SMART-Seq v4 Ultra Low Input RNA kit, continued from the tagmentation step. Library preparation was performed using a Nextera XT DNA Sample Preparation Kit (Illumina) as described in the protocol. Following library preparation, samples were pooled and sequenced using a NextSeq 300 high-output kit in an Illumina NextSeq 500 System with $2 \times 150$ paired-end reads.

\section{Processing of RNA Sequencing Data}

After sequencing, raw reads were de-multiplexed and preprocessed using Trimmomatic and Flexbar. Then, raw sequencing reads were aligned to the Ensembl Drosophila ananassae genome (release 37), using a STAR aligner with the following parameters: trimLeft $=10$, minTailQuality $=15$, minAverageQuality $=20$, and minReadLength $=30$. Gene counts were calculated using HTSeq. For convenience, Ensembl gene IDs use the corresponding gtf file (ftp://ftp.ensemblgenomes.org/pub/metazoa/release-37/gtf/drosophila_ananassae/Drosophila_ananassae.dana_caf1.37.gtf.gz) as a reference.

\section{Bioinformatical Analysis of RNA Sequencing Data}

To assess differential expression, we utilized DESeq2. To account for technical dropouts, we first used zingeR to zeroWeightsLS function to weigh gene expression against dropouts. We then used DESeq 2 to normalize cell gene expressions and then run a differential gene expression analysis.

\section{Bioinformatical Analysis of the D. ananassae Comparative}

Transcriptome Data

Transmembrane protein structures were predicted using the MemBrain online webserver [27]. As there is no information available concerning the $D$. ananassae genes and proteins, we used the www.flybase.org database to get knowledge about their D. melanogaster orthologs. Gene Ontology (GO) enrichment analysis was performed using GO: TermFinder open source software [28]. Enrichment analysis was performed against the background of all of the annotated $D$. melanogaster genes on www.flybase.org database. Results were summarized and visualized with REVIGO [29]. Physical interaction networks were constructed with the Cytoscape software [30], using interactions listed on the www.flybase.org database.

\section{Results}

\section{Ultrastructural Features of MGH Development}

Prominent features of developing MGHs could be noted as early as $24 \mathrm{~h}(24 \mathrm{~h})$ after $L$. boulardi parasitoid wasp infection. These included differentiation of cells larger than plasmatocytes, and appearance of light vesicles as a rim in these cells (Fig. 1a). At $48 \mathrm{~h}$, differentiating cells displayed more substantial size growth and changed their originally oval shape to spindle. In addition, cellular branching, multiple nuclei, and several light vesicles in large areas of the cytoplasm become increasingly apparent (Fig. 1b). By $72 \mathrm{~h}$, multinucleated cells fully differentiated into MGHs; they became enormously large and strikingly different from plasmatocytes (Fig. 1c). The giant hemocytes also attached to the surface of parasitoid and extended projections under its chorion (Fig. 1d). Furthermore, they grew cytoplasmic extensions, which intruded into the intersegmental grooves of the parasitoid (online suppl. Fig. S1a; for all online suppl. material, see www.karger.com/doi/10.1159/000520110). At some sites, layers of MGHs wrapped the parasitoid, forming a capsule (online suppl. Fig. S1b). In addition, dense vesicles also appeared in the cytoplasm of MGHs. Both the newly formed light and dense core vesicles were associated with the Golgi apparatus (Fig. 1e, f). Microtubules could also be frequently seen closely aligned along linear rows of dense vesicles, many of which seem to have fused with each other in an elongated shape (Fig. 1g).

Mature MGHs had unique ultrastructural features, which clearly distinguished them from other known cell types. Their cytoplasm was divided into 2 structurally different regions: a basal part, adjacent to the parasitoid, and an apical area, opened to the hemocoel. The thickness of both regions, as well as that of the MGH itself, was highly variable (Fig. 2). The basal region showed a characteristic abundance of dense bodies (see next paragraph), whereas the apical region contained cell nuclei and perinuclear areas that gave rise to projections into the hemolymph. Of note, the perinuclear areas were the most similar to a typical cell structure in arrangement and organellar composition. In certain locations, a high number of lipid droplets could also be detected (Fig. 2). Finally, the plasma membrane of apical regions irregularly invaginated into the cytoplasm, which created a spongy, labyrinthlike structure (Fig. 2, insert of Fig. 2, 3d).

\section{Formation of a Special Multiform Dense Body System}

As noted above, basal cytoplasmic regions were characterized by the abundance of dense bodies (Fig. 2, 3), which formed already $48 \mathrm{~h}$ after the parasitoid wasp infection and later aligned along microtubules, finally forming a fused, elongated structure (Fig. 1g). These pointed toward the basal membrane and frequently appeared as a complex multiform network, consisting of vesicular, tubular, lamellar, and irregularly shaped bulky structures 

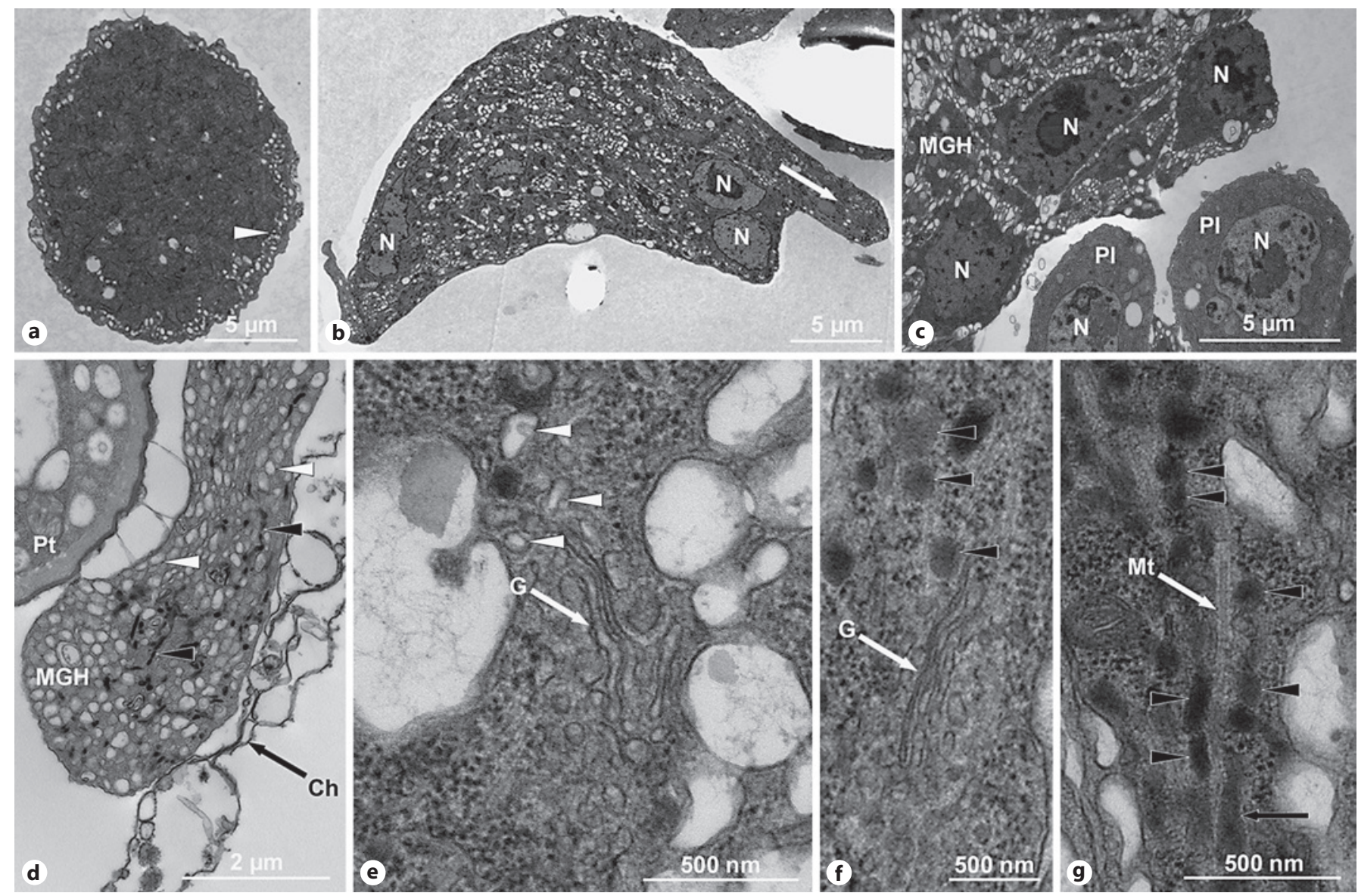

Fig. 1. Ultrastructural features of MGH differentiation following L. boulardi parasitoid wasp infection. a Twenty-four hours following wasp infection. In MGH precursors, light vesicles appeared around the cell periphery (white arrowhead). b Forty-eight hours after parasitization MGHs display increased size and nuclear (N) number, branching begins (white arrow), and a large number of vesicles throughout the cytoplasm. c Seventy-two hours following parasitization. Higher magnification image shows a part of an MGH (in the upper left corner), with 3 nuclei (N) and many vesicles. The MGH is clearly distinguishable from nearby plasmato-

cytes ( $\mathrm{Pl}$, in the lower right corner) by size and structure. $\mathbf{d}$ Dense (black arrowheads) and light (white arrowheads) vesicles in an $\mathrm{MGH}$ extend on the surface of the parasitoid (Pt) under the chorion $(\mathrm{Ch})$. e Image shows the emergence of light vesicles (white arrowheads) from the Golgi apparatus (G). f Image shows the emergence of dense vesicles (black arrowheads) from the Golgi apparatus (G). g Dense vesicles (black arrowheads) are aligned along microtubules (Mt) and form a fused, elongated vesicle (black arrow). e-g Images were taken at $48 \mathrm{~h}$ after parasitoid infection.

(Fig. 2, 3). Although with variable thickness, this multiform dense body system (MDBS) represented a continuous lamellar layer adjacent to the surface of parasite (Fig. 2, 3a, b, c). Analysis of multiple samples revealed that some MDBSs were also present in deeper apical regions of the cytoplasm (Fig. 3d).

\section{Recognition of Acidic Vesicles in the MGHs}

To further characterize MDBSs, we used the LysoTracker dye, a tracer for acidic organelles, and carried out a comparative staining of MGHs and plasmatocytes. We found numerous LysoTracker positive structures in both

the MGHs and the plasmatocytes. However, there was a significant difference in dye retention time, which was $>2$ min in plasmatocytes, but $<2$ min in MGHs (online suppl. Fig. S2). In MGHs, accretions of the dye appeared basally, in well-recognizable continuous areas in contact with the parasite (Fig. 4). In addition, scattered dye staining could also be seen in the apical region, sometimes close to the apical surface, either as larger particles or as a fine network with high- or low-intensity staining, respectively. This observation was reminiscent of the electron microscopy analysis, which also showed some MDBS-rich areas in apical regions (Fig. 3d). This remarkable similarity in 
Fig. 2. A polarized region of mature MGH attached to the parasitoid wasp $(\mathrm{Pt})$. The basal region $(\mathrm{Ba})$ on the surface of the invader; the opposite apical side (Ap) with projections (Pr) into the hemolymph. The thickness of basal and apical regions is variable. A multiform dense body system (MDBS) appears in the basal region, consisting of a loose dense network (white arrowheads) and a continuous thin dense layer (black arrowheads) on the cuticle (Ct) of the parasitoid. Spongy canalicular labyrinth opens to the hemolymph (arrows), a similar structure in the inset at a higher magnification. The middle region of the cell is rich in lipid droplets (L), whereas nuclei $(\mathrm{N})$ are localized in the apical region.

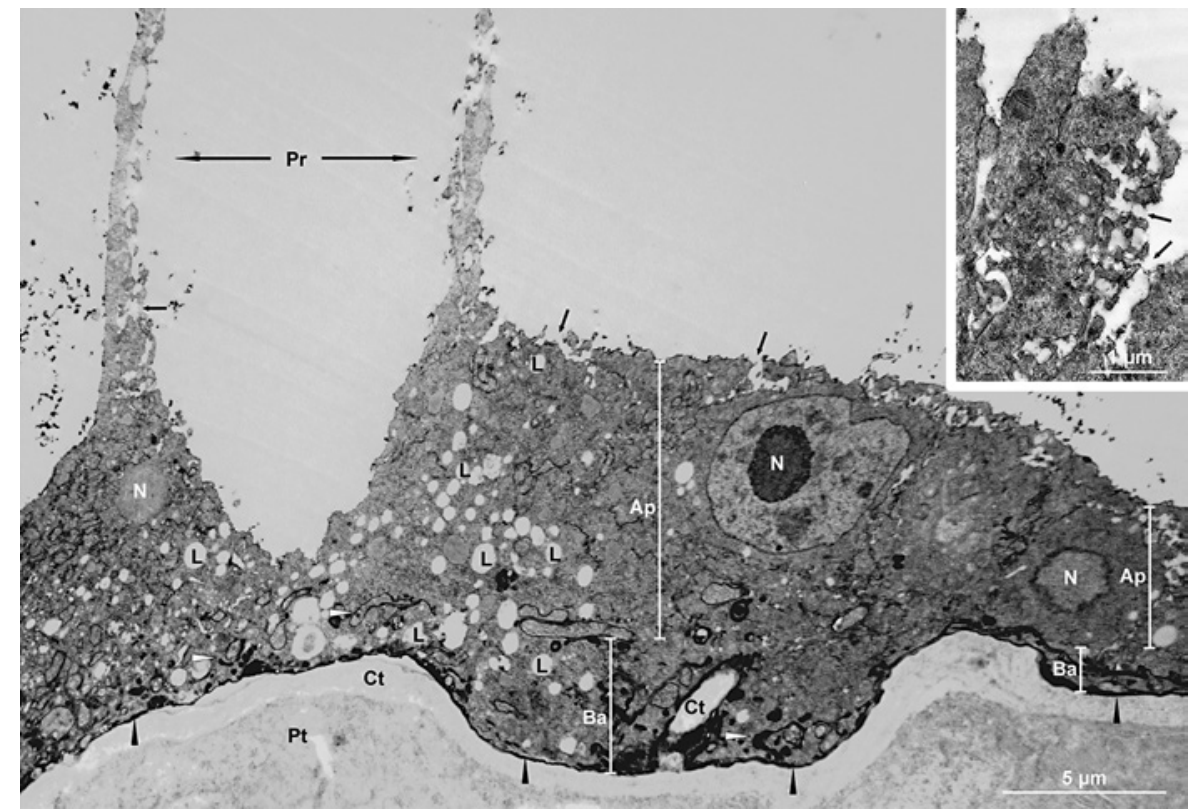

the localization and shape of LysoTracker positive structures and MDBs in MGHs suggested that these structures represent overlapping subcellular elements. In phagocytic cells, LysoTracker positive acidic vesicles and structures appeared as lysosomes. However, MGHs are nonphagocytic, and the complex morphology of MGH MDBSs differed from the lysosomal apparatus described in other cell types. To better understand this distinction, we looked for ultrastructural signs of degradative activity in MDBS. The fine vesicular, tubular, and sheet-like elements appeared as a homogeneous dense matter (Fig. 3) and did not seem to be engaged in degradation. Instead, they resembled primary lysosomes, which were also dense, homogeneous, and contained lysosomal enzymes, primed to be activated by material destined for digestion [31]. Digestion has been shown to lead to morphological deterioration, making the lysosomal content heterogeneous and morphologically unrecognizable [32], frequently referred to as "intracellular garbage bags" [33, 34]. Indeed, in our electron microscopy sections, the MDBS system regularly appeared as heterogeneous and seemingly disorganized (Fig. 3a, d), similar to the classical view of digestive lysosomes. Without heterophagic input, the only means for MDBSs to get access to degradable substrate is autophagy. In support of this possibility, we found autolysosomes and autophagosomes in large quantities in MGHs, and most frequently in MDBS-rich area (Fig. 3a; online suppl. Fig. S3). To summarize, our confo$\mathrm{cal}$ and electron microscopy data support the notion that
LysoTracker positive structures and MDBSs represented a lysosomal system in MGHs, specifically involved in selfdegradation of cytoplasmic portions by autophagy.

\section{Light Vesicles Develop into an Elaborate Reticular \\ System}

In mature MGHs, light vesicles remain the most conspicuous organelles that tend to be organized in areas, where they become disproportionately abundant. The expansion of the light vesicles appeared to take place in 2 major steps. As alluded above, the first step is characterized by accumulation of individual vesicles. They were either close to the cell periphery (Fig. 1a) or scattered more or less evenly in the cytoplasm (Fig. 1b-d). In areas closer to the nucleus, they often intruded into the perinuclear area, rendering the normal cytoplasm island-like (Fig. 1b, c, 5a). In the second step, medium-sized light vesicles partially fuse into a segmented reticular network, in which individual units became interconnected yet discernible (Fig. 5). In this manner, rows, or sheets in 3 dimensions, of a light reticular system (LRS) became apparent. Then, the medium-sized units of the LRS grew in size and became larger and flat (Fig. 5a), taking up roundish or irregular shapes, and completely occupying extensive areas of the MGH cytoplasm (Fig. 5b). This rapid growth presumably involved both fusion and new membrane synthesis. Finally, LRS sheets were either directly adjacent to each other or separated only by a very thin layer of ground cytoplasm (Fig. 5a, b). 
Fig. 3. Organization of the multiform dense body system (MDBS) in the MGHs. a Image shows MDBS elements with vesicular (Ve), tubular (Tu), lamellar (La), and bulky $\mathrm{Bu}$ ) structures adjacent to the cuticle $(\mathrm{Ct})$ of the parasitoid. $\mathbf{b}$ Continuous dense layer with variable thickness covering the cuticle (Ct) of the parasitoid. c Higher magnification image shows the lamellar structure of the dense layer covering the cuticle of the parasitoid $(\mathrm{Ct})$. d Image shows the complexity of MDBS in the deeper apical region of an MGH: dense homogeneous vesicles $(\mathrm{Ve})$, tubules $(\mathrm{Tu})$, lamellae (La), and heterogeneous bulky $(\mathrm{Bu})$ elements with lighter areas. The apical part of MGH displays a spongy structure $(\mathrm{Sp})$.
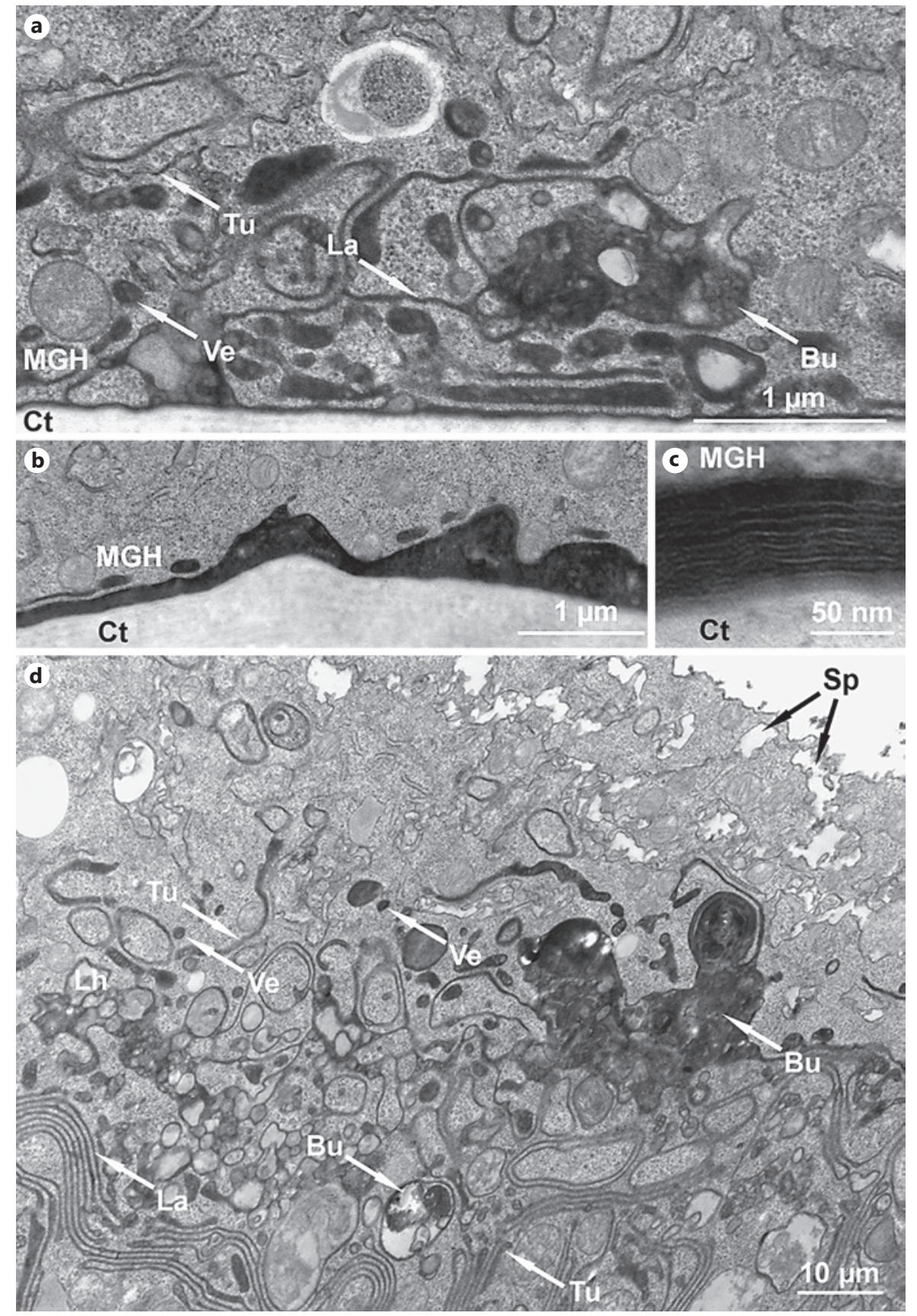

Within LRSs, only a faint, less electron-dense, inhomogeneous, and diffuse material was detected (Fig. 5a), which appeared as a fluffy precipitate in the large cisternal elements (Fig. 5b). The largest cisternal spaces contained a considerable amount of precipitate, which accumulated under the chorion of the parasitoid, presumably to be discharged into the hemolymph (online suppl. Fig. S4).
Comparative Morphology and

Transcriptome of MGHs

\section{Formation of Giant Cell Exosomes}

We also found that smaller apical surface areas of MGHs developed into a loose network of cytoplasmic extensions (Fig. 6). These extensions frequently emerged from perinuclear areas that were rich in RER, mitochondria, polysomes, and dense bodies (Fig. 6b, c). In addition, MGHs carried outgrowths of microfilaments, and a high number of very small vesicles, which we named 
Fig. 4. Multinucleated giant hemocytes forming the capsule $(\mathrm{Ca})$ around the parasitoid (Pt) generate thin flat LysoTracker positive areas (white arrows in the right image) along the attachment site with the parasitoid. Images are visualized with an Olympus FV1000 confocal LSM microscope.
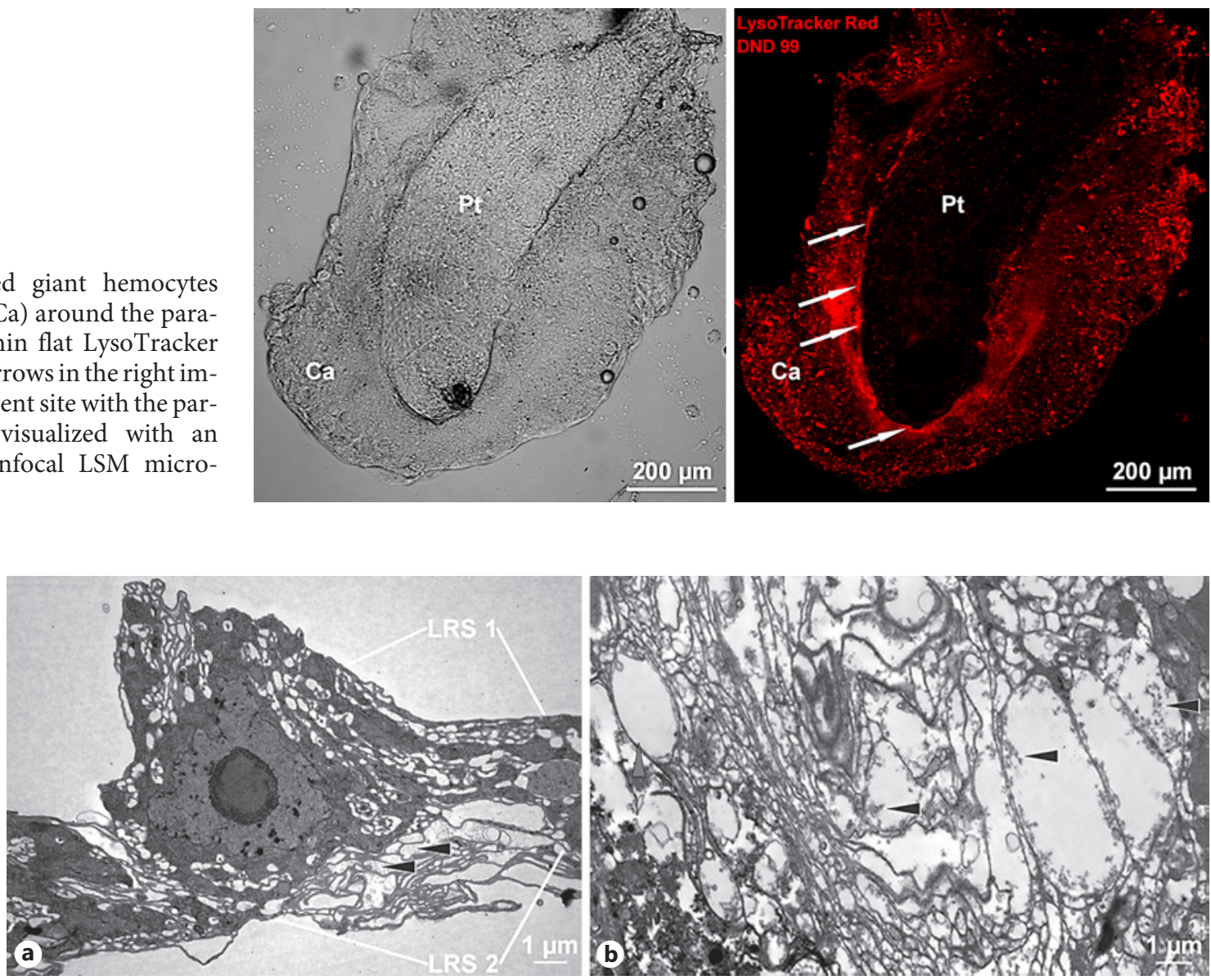

Fig. 5. Development of the light reticular system (LRS). a Segmented network with medium-sized units (LRS1). Expansion into large flat units (LRS2). Faint diffuse material can be seen in the lumen (arrowheads). b Further expansion of adjacent LRS units with variable size and shape very close to each other. Precipitated content of the large cisternal elements (arrowheads).

GCEs. Based on morphological traits, GCE originated from the cell surface by budding and/or detaching from the tip of the filaments. It was unlikely that GCEs originated from unspecific cellular disintegration, as various intracellular organelles, the polysomes and the mitochondria, which are the most vulnerable to deleterious effects, were intact.

\section{Rapid Plasma Membrane Development in the MGHs}

To further study the ultrastucture of the MGHs, we performed immunostaining with the 7C5 antibody, which we previously characterized as a specific marker for MGHs [12]. Immuno-transmission electron microscopic analysis revealed that 7C5 strongly and specifically reacted with the membrane of MGHs (Fig. 7a) and revealed a special ultrastructural arrangement of the plasma membrane. To underscore the notion of specificity, the membranes of nearby plasmatocytes were also negative (Fig. 7b). In MGHs, robust staining was seen on budding exosomes (Fig. $7 \mathrm{a}, \mathrm{c}, \mathrm{d}, \mathrm{e}$ ) and on the invaginated labyrinths in spongy areas of the MGH surface (Fig. 2, 3d). Since most, but not all, membranes of the spongy structure were immunostained, we presume that in addition to the plasma membrane invaginations, intracellular canals were also present in this area (Fig. 7c, e). Further, membranes of the large vacuoles were negative (Fig. 7c, d), suggesting that their enclosure was different from the plasma membrane and thus they likely represented intracellular compartments. Interestingly, however, some intracellular membranes also appeared as 7C5 positive. These were fine vesicular, tubular, and cisternal elements (Fig. 7d), possibly com- 

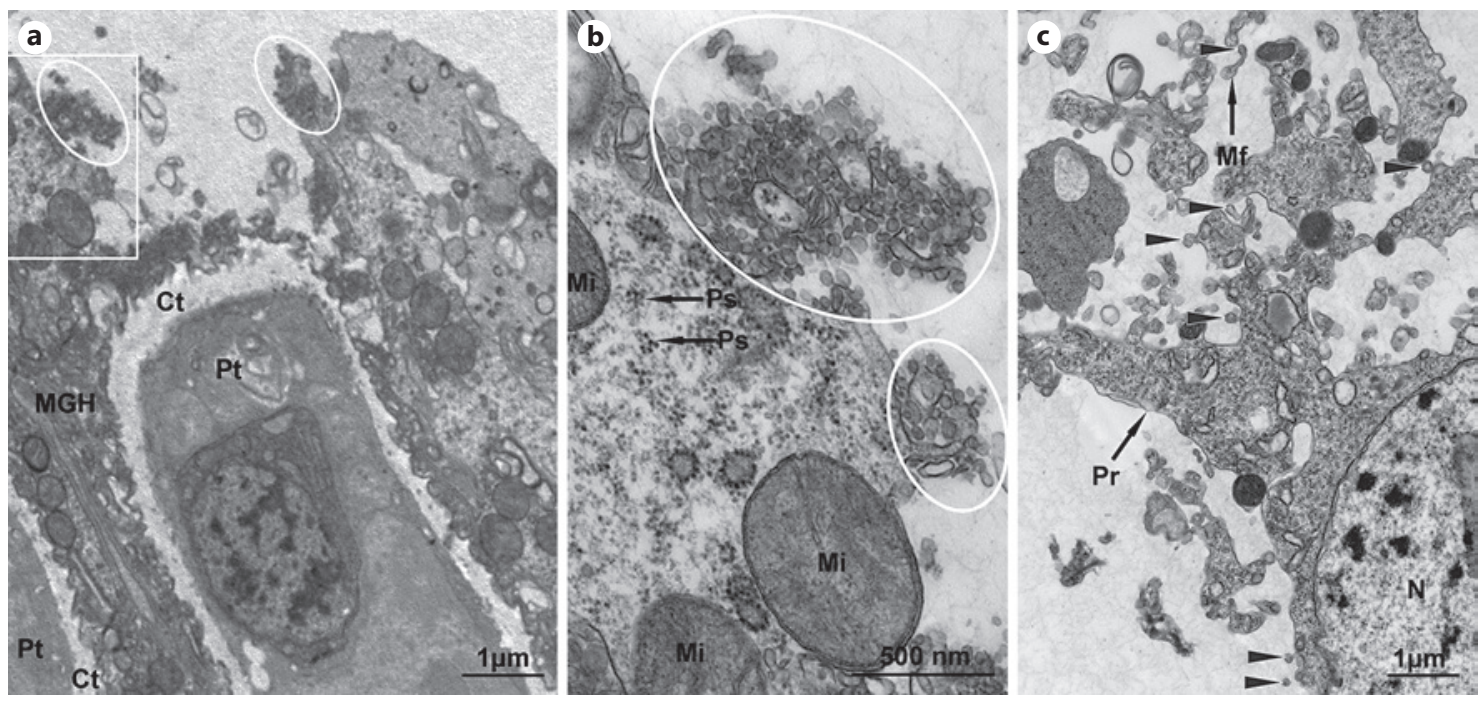

Fig. 6. Giant cell exosome (GCE)-generating areas in MGH. a MGH settled on the cuticle (Ct) of the parasitoid (Pt). GCE formation sites are circled. $\mathbf{b}$ (Enlarged part from a) GCE formation sites (circled). Polysomes (Ps) and mitochondria (Mi) appear intact and undamaged. c GCE generation by apparent microbudding (arrowheads). $\mathrm{N}$, nucleus; Pr, projection; Mf, microfilament.

ponents of the post-Golgi area. We presume that these 7C5 antigen-expressing membrane elements were generated for further plasma membrane growth and projection formation (Fig. 7e). This scenario was supported by the observation that the plasma membrane of newly developed MGH projections was apparently formed with the fusion of 7C5 positive vesicular membranes (Fig. 7e).

\section{Transcriptomic Analysis of Differentiated MGHs}

Our above results have already revealed a highly dynamic picture of MGH development and parasite encapsulation. We hypothesized that these processes were supported by increased transcriptional activity and gene expression changes. To test this hypothesis, we isolated MGHs by electrophysiological patch-pipettes $72 \mathrm{~h}$ after L. boulardi infection and studied their transcriptomic content by mRNA sequencing. For comparison, we also isolated and sequenced the mRNA of activated plasmatocytes nearby. For each type, 5 single cells were pooled per sample and 5 samples of each were collected for analysis. In total, we detected 9,106 different gene transcripts. Results obtained after normalization showed that 705 genes were differentially expressed between MGHs and plasmatocytes. Of these, 481 and 224 genes were expressed at significantly higher and lower levels, respectively, in MGHs than activated plas- matocytes (online suppl. Table S1). These also included 200 and 8 genes that were detected exclusively in MGHs and plasmatocytes, respectively. Further, we detected 78 D. ananassae genes (within the 705 differentially expressed genes), which did not have D. melanogaster orthologs (online suppl. Table S1). Of these, 63 were expressed at significantly higher and 15 at significantly lower level in the MGHs than the activated plasmatocytes.

To gain insights into possible functional interactions, all putative proteins encoded by the 705 differentially expressed genes were further analyzed in silico. Because to date no available information on D. ananassae proteins existed, we used the www.flybase.org database to analyze $D$. melanogaster protein orthologs. GO enrichment analysis did not reveal any "biological processes" that would be shared by MGHs and plasmatocytes (online suppl. Fig. S5). In MGHs, most genes were related to cell communication, signaling, vesicle, and membrane function. By contrast, genes significantly higher expressed in plasmatocytes were associated with metabolic processes. Subsequently, we used GO "cellular component" analysis to illuminate the possible subcellular localization of proteins. This revealed that $\mathrm{MGH}$-enriched genes encoded proteins that localized in membranes, vesicles, vacuoles, lysosomes, and SNARE complexes, a family of proteins involved in ves- 

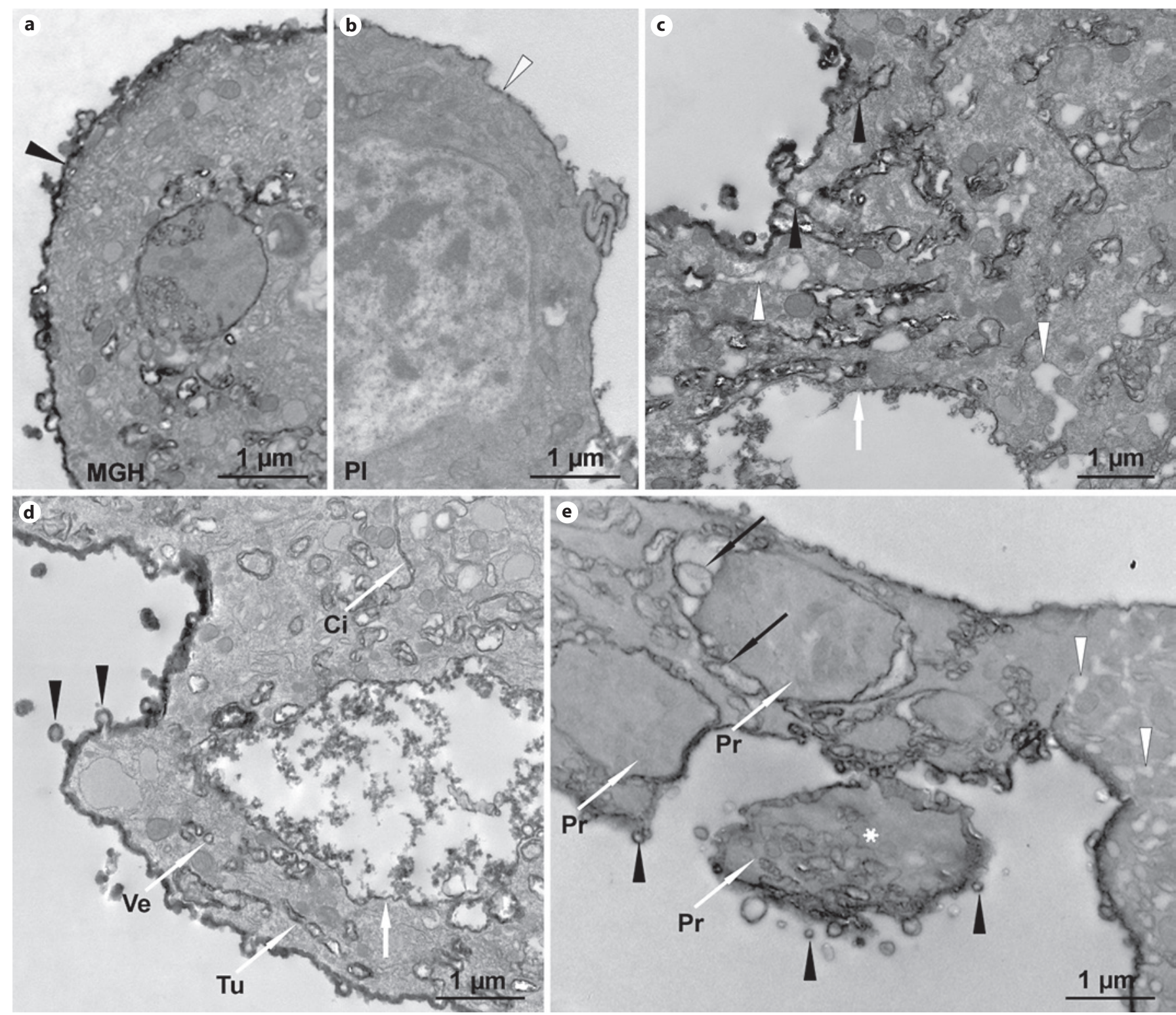

Fig. 7. Plasma membrane development in the MGHs revealed by immuno-transmission electron microscopy. a MGH plasma membrane shows strong 7C5 staining (black arrowhead). b Plasmatocyte plasma membranes lack 7C5 staining (white arrowhead). c 7C5 positive plasma membrane invaginations (black arrowheads) and 7C5 negative intracellular canals (white arrowhead). Note the lack of labeling on large intracellular vacuole membranes (white arrow). d Strong 7C5 labeling of the plasma membrane on spo-

radic budding exosomes (black arrowheads), and intracellular fine vesicular $(\mathrm{Ve})$, tubular $(\mathrm{Tu})$, and cisternal $(\mathrm{Ci})$ structures. Note the lack of labeling on the membrane of the large vacuole (white arrow). e Vesicles possessing 7C5 positive membranes (black arrows) fuse to form the plasma membrane of the $\mathrm{MGH}$ projections (Pr). Asterisk labels a newly developed projection detaching from the cell body. Intracellular canals (white arrowheads) and giant cell exosomes (black arrowheads) are 7C5 negative.

icle fusion [34]. By contrast, plasmatocyte-enriched genes frequently encoded proteins that localized in cytosol, nucleus, and different subcellular organelles, such as mitochondria, ribosomes, and the endoplasmic reticulum (online suppl. Fig. S6).

Transcriptional Signatures of MGH Development and Function

Next, we sought to gain insights into gene expression changes that could contribute to the rapid differentiation and development of MGHs after parasitoid wasp infec- 
Fig. 8. Venn diagram shows the result of cross-comparison analysis of the genes up(a) and downregulated (b) in the MGHs compared to uninduced blood cells (blue), activated plasmatocytes compared to uninduced blood cells (red), and MGHs compared to activated plasmatocytes (green) of $D$. ananassae. The list of the respective genes is presented in online suppl. Table S4. Both comparisons, as expected, contain a null (0) set of genes, as we found no genes that we simultaneously upregulated in MGHs versus activated plasmatocytes, and activated plasmatocytes versus uninduced blood cells, but not upregulated in MGHs versus uninduced blood cells.

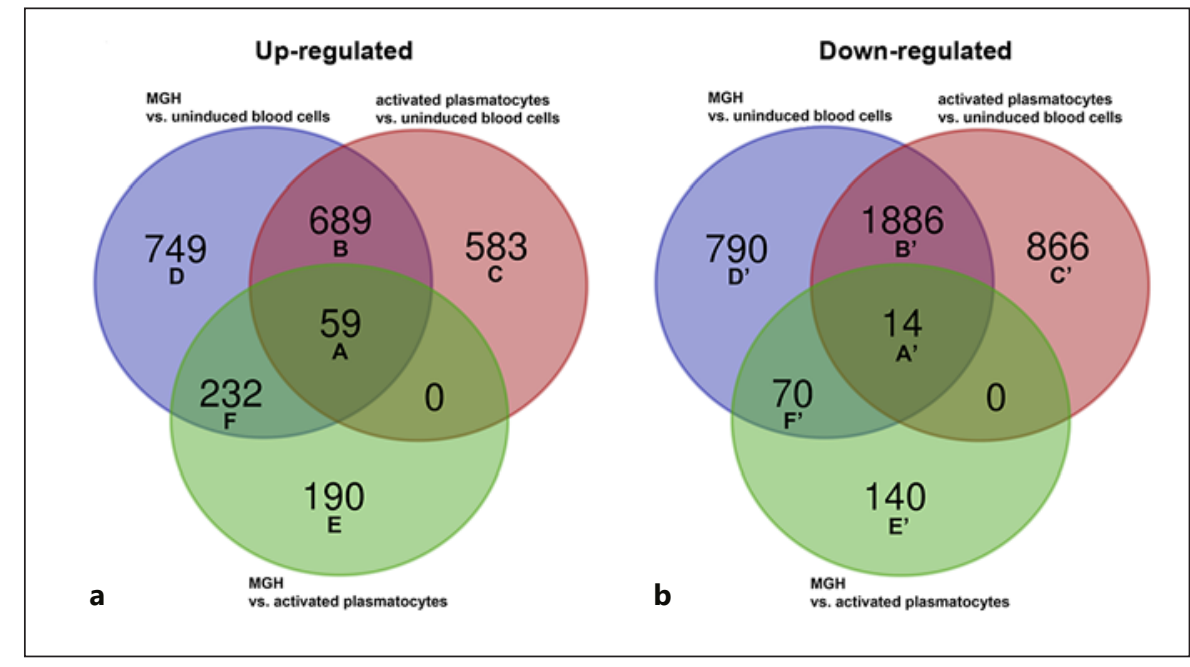

tion. For this, we complemented our data sets with mRNA sequencing from uninduced blood cells, which would give rise to MGHs after infection. Because uninduced blood cells represent a population of heterogeneous plasmatocytes, we isolated mRNA for transcriptome analysis from 5 independent and uninduced blood cell pools from third instar larvae. For comparability, the age of these larvae was identical to that of the wasp-infected larvae. Then, we compared the transcriptomes of uninduced blood cells and MGHs in a similar fashion as presented above for activated plasmatocytes and MGHs.

We found a total of 4,489 genes that were differentially expressed: 1,729 and 2,760 genes were significantly higher expressed in MGHs and uninduced blood cells, respectively (online suppl. Table S2). Among these, 24 and 1,495 genes were expressed exclusively in MGHs and uninduced blood cells, respectively. We also found $612 \mathrm{D}$. ananassae genes (within the 4,489 differentially expressed genes), which did not have known D. melanogaster orthologs (see online suppl. Table S2). Of the 612 genes, 167 and 445 genes were significantly higher expressed in MGHs and uninduced blood cells, respectively. We then further analyzed all 4,489 differentially expressed genes in silico (online suppl. Table S2). GO analysis for "biological process" revealed that MGHs mostly expressed genes, whose protein products were involved in vacuolar, vesicular, endosomal processes, cellular secretion, microtubule-based transport, ATP synthesis, and autophagy. By contrast, the uninduced blood cells expressed more transcripts whose protein products were relevant for basic cellular processes, such as carbohydrate-, lipid-, and protein-metabolism, catabolism, and also developmental processes (online suppl. Fig. S7). Further, GO enrichment analysis in "cellular component" category revealed that MGH-enriched genes encoded proteins that localized in membranes, vesicles, lysosomes, SNARE complexes, vacuolar-type ATPase complexes, endosomal sorting complex required for transport, cytoskeleton, Golgi apparatus, and mitochondrion (see online suppl. Fig. S8). By contrast, most uninduced blood cell-enriched genes encoded proteins that localized mainly in the cytosol, peroxisomes, ribosomes, nucleus, and other intracellular organelles (online suppl. Fig. S8).

Finally, we asked which gene expression changes characterized MGHs and activated plasmatocytes, commonly or uniquely, after parasitic infection. For this, we considered genes that were significantly up- and downregulated in (i) MGHs compared to uninduced blood cells, (ii) activated plasmatocytes compared to uninduced blood cells, and (iii) MGHs compared to activated plasmatocytes (Fig. 8; online suppl. Tables S1-S4). We found 748 $(689+59)$ genes, which were upregulated in MGHs and activated plasmatocytes compared to uninduced blood cells (Fig. 8a). These likely represent genes that were commonly activated by the infection. Of these, 59 genes were further enriched in MGHs compared to activated plasmatocytes, suggesting differences in how these genes were utilized by the 2 cell types. Further, we found 749 and 583 genes, which were upregulated only in MGHs and activated plasmatocytes, respectively, compared to uninduced blood cells. These presumably represented separate transcriptomic responses induced by infection. Finally, we found 232 and 190 genes that were enriched in MGHs either compared to both uninduced blood cells and activated plasmatocytes or to activated plasmatocytes only, respectively. These, together with the previously 
mentioned 749 genes, were likely responsible for creating the unique makeup of MGHs. In addition to upregulation, gene downregulation could also contribute to immune responses mediated by MGHs and activated plasmatocytes. Therefore, we separately analyzed downregulated genes in each comparison (Fig. 8b; note that downregulation in the $\mathrm{MGH}$ vs. uninduced blood cell comparison, used as an example, would be equivalent to upregulation in the reverse, uninduced blood cell vs. $\mathrm{MGH}$, comparison). This analysis revealed that a larger number of genes were downregulated $(1,886+14$; Fig. $8 \mathrm{~b})$ than upregulated $(689+59$; Fig. $8 \mathrm{a})$ in $\mathrm{MGH}$ and activated plasmatocytes in response to parasitoid infection. However, there were more genes that differentiated MGHs by upregulation (232; Fig. 8a) than downregulation (70; Fig. 8b) when compared to both other cell types. The large number of genes, overexpressed in the MGHs, based on their D. melanogaster orthologs and the available data on www.flybase.org, arranged in several groups, which were relevant for the composition and function of these highly motile cells, are abundant in vesicles, vacuoles, lysosomes, endosomes, and lipid droplets as observed by the electron microscopic analysis (Table 1).

\section{Protein Interaction Networks Encoded by MGH- \\ Enriched Genes}

Next, as a proxy of potentially MGH-specific function, we were interested in how the MGH-enriched genes govern protein interactions. Since there was no available information regarding $D$. ananassae proteins, we used available interaction data of their D. melanogaster orthologs, based on the www.flybase.org database. Using this database, we constructed physical interaction networks from proteins encoded by genes that were enriched in MGHs compared to both uninfected blood cells and activated plasmatocytes. This analysis revealed several significantly enriched clusters, which were separated by biological processes and in which proteins may form stable complexes or associate transiently. To organize the different clusters, we focused on the major categories, such as vesicles, Golgi apparatus, exocytosis, and cytoskeleton (Fig. 9-11). We chose these categories in part because the largest clusters belonged to these categories, and in part also because our electron microscopic analyses revealed enhanced cellular activity in these areas. In this confirmatory analysis, we tested if there was a correspondence between the significant gene clusters and structural observations. In the vesicle category, we were able to enlist different clusters that were associated with vesicle-, vacuole-, lysosome-, autophagy, and lipid storage-related clusters
(Fig. 9). These corresponded to our findings made by electron microscopic analysis, which revealed abundant presence of these cellular structures in MGHs (Fig. 1, 2, $4,5)$. Next, we found significantly enriched clusters that were related to Golgi apparatus and exocytosis (Fig. 10). These again corresponded to our findings made by electron microscopic analysis, which revealed vesicle accumulation and budding on the cell surface. In the structural category, we enlisted significant gene clusters that were associated with cytoskeletal organization, motility, and adhesion (Fig. 11). Although the appearance of these functions was not directly supported by structural analysis, they were consistent with a model, in which active cytoskeletal rearrangements in MGHs support growth, adhesion, and the encapsulation reaction.

\section{Comparison of the Gene Expression Profile of MGHs and Lamellocytes}

Finally, to further pursue the question regarding the identity of encapsulating mechanisms, we compared the molecular make-up of encapsulating blood cells in different species of Drosophilidae. Specifically, based on previous studies, we carried out a comparison of the lamellocyte-specific genes and proteins that were characterized in D. melanogaster [35-40] and tested if these were also enriched in MGHs and/or activated plasmatocytes of $D$. ananassae. We found that 61 and 33 D. melanogaster lamellocyte-specific genes were also selectively expressed in D. ananassae MGHs and in the activated plasmatocytes, respectively (online suppl. Table S5). In the following, we will refer to molecules by their gene name in D. melanogaster, without specifying the name of their orthologs in D. ananassae, which can be found in online suppl. Table S5. Shared genes included the glucose-producing Trehalase and the glucose transporter-encoding CG1208, the presence of which would be consistent with a high energy demand associated with immune activation, maturation, and/or encapsulation in both lamellocytes and MGHs. Moreover, actin-interacting proteins (e.g., Actin 42A, Annexin B9, Jupiter, the filopodia/lamellipodia-associated Twinstar, Parvin, Actin-related protein 3, Pod1, Flare, Rho GTPase-activating protein at $18 \mathrm{~B}$, and the Myosin7 a binding protein encoded by CG43340) presumed to be required for encapsulation and cytoskeletal rearrangements were highly enriched in both cell types. Furthermore, we found shared molecules, which are involved in cell-adhesive interactions and formation of cell-cell junctions, and thus likely important for the encapsulation. Our data showed that integrin beta-nu subunit (itgbn), rhea, Rac2, taxi, FER tyrosine kinase, and bves were also 
Table 1. Predicted function of the $D$. ananassae genes overexpressed in the MGHs

Predicted function of genes expressed at a significantly high level in the MGHs. D. melanogaster orthologs are listed.

Vesicle-related

14-3-3zeta, Ack, alc, alphaSnap, AnxB9, AP-2mu, AP-2sigma, Appl, Arf102F, Arf51F, Arf79F, Arl1, Arl2, Arl4, Arl5, Arl8, Atet, Atg6, ATP6AP2, awd, bchs, Bet1, Bet3, Bet5, BicD, Blos1, boca, BORCS5, CanB2, car, CASK, Ccz1, Cdc42, CG10103, CG10435, CG13531, CG15012, CG16865, CG18659, CG30423, CG32069, CG32576, CG33635, CG43322, CG4645, CG5021, CG5104, CG5510, CG7956, CG8134, CG8155, CG9067, Chc, Chmp1, CHMP2B, Clc, cni, comm, Dab, DCTN2-p50, Dlc90F, epsilonCOP, Esyt2, Exo70, Exo84, fab1, Flo1, Flo2, fwe, gammaSnap1, Gdi, Gga, gish, Gos28, Gp150, IdlCp, Lerp, Isn, It, Madm, Membrin, milt, Mon1, Muted, nudE, or, PAPLA1, Past1, Pi3K59F, Pldn, poe, Ppt1, ps, Rab1, Rab11, Rab18, Rab19, Rab2, Rab21, Rab35, Rab39, Rab40, Rab5, Rab7, Rab8, RabX1, RabX6, Rac2, Rap1, Ras64B, Rep, Rho1, Rich, Rint1, Rop, Sec10, Sec15, Sec5, Sec6, shi, shrb, sing, Snap24, Snap29, Snx16, spir, spri, stac, stmA, strat, Syb, Synd, Syngr, Syt1, Syx13, Syx16, Syx17, Syx1A, Syx4, Syx5, Syx7, Syx8, Thor, Tomosyn, Trs20, Trs23, Trs31, Trs33, TSG101, unc-104, Use1, Uvrag, Vamp7, Vap33, Vha16-1, VhaAC45, Vps11, Vps15, Vps16A, Vps2, Vps20, Vps24, Vps25, Vps28, Vps36, Vps37B, Vps39, Vps60, Vta1, Vti1b, WASp, yki, Ykt6, zetaCOP

Lysosome-related

Akap200, Arf79F, Arl8, asrij, Atg8a, ATP6AP2, bchs, Blos1, Blos2, Blos3, Blos4, BORCS5, BORCS6, car, Ccz1, cd, cer, CG10681, CG14184, CG14977, CG32225, CG32590, CG4080, CG4847, CG6707, CG7523, CHMP2B, CIC-b, comm, Cp1, CREG, CtsF, ema, fab1, FIG4, GILT2, Iml1, Lamtor5, Lerp, LManll, It, Mon1, Muted, Nprl3, Nup44A, or, Pldn, Ppt1, Ppt2, prd1, Psn, Rab2, Rab7, RagA-B, RagC-D, Rilpl, Sap-r, Snx16, stac, Syx16, Syx17, Syx1A, Trpml, Vamp7, Vha13, Vha16-1, Vps11, Vps16A, Vps16B, Vps25, Vps36, Vps60, wash, yki

Lipid metabolism

ABCA, Ack, alpha-Est7, AnxB10, AnxB11, AnxB9, Aps, Arf79F, Arfip, Atg12, Atg7, Atg8a, awd, bchs, beta4GalNAcTB, brn, Cerk, CG11975, CG14883, CG15629, CG17544, CG1941, CG1946, CG31460, CG31683, CG31717, CG31935, CG3246, CG33116, CG33774, CG9743, Dad1, Dgat2, Dgkepsilon, disp, Eato, egh, Esyt2, fab1, FER, fh, firl, ghi, gny, Hex-A, Hsl, iPLA2-VIA, kud, lace, lace, Ldsdh1, LPCAT, Npc2a, ORMDL, Pgi, Pi3K59F, PIG-V, PTPMT1, Rab18, Rac2, Rheb, RhoGAP92B, Sap-r, scramb1, smt3, SNF4Agamma, St1, Start1, subdued, sws, Synd, Syt1, TMS1, Treh, Ubc2, Vha16-1, vib, Vps36, zetaCOP

Cytoskeletal organization, motility

14-3-3epsilon, 14-3-3zeta, Ack, Act42A, Akap200, ALiX, alpha-Cat, aPKC, Arf51F, Arfip, Arl2, Arp1, Arp10, Arp2, Arp3, Arpc1, Arpc2, Arpc3A, Arpc3B, Arpc4, Arpc5, awd, bchs, betaTub56D, BicD, BORCS5, BRWD3, Bsg, Calx, Cam, CASK, CCDC53, Cdc42, Cdk9, Ced-12, CG10984, CG13366, CG15701, CG18190, CG1890, CG31715, CG32264, CG32590, CG43867, CG4537, CG6891, CG7497, CG8134, CG9288, Chc, chic, cib, cindr, cnn, cpa, cpb, Crk, dah, DCTN2-p50, DCTN3-p24, DCTN4-p62, DCTN5-p25, DCTN6-p27, Dhc16F, Diap1, Dlc90F, Dlic, Doa, drk, egh, egl, Ehbp1, FER, flr, form3, FRG1, Ggamma1, gish, GMF, grk, gukh, Hem, HSPC300, insc, jub, Jupiter, Kap3, Klc, Klp64D, Ids, mad2, mago, Mer, mgr, milt, Mlc-c, Mob4, moody, msn, mtm, nod, nudE, Nup44A, par-1, parvin, pigs, PIP4K, pnut, pod1, Psn, Pvr, Rab1, Rab11, Rab21, Rab35, Rac2, Rbp, Rcd5, ReepA, rhea, Rho1, RhoGAP18B, RhoGAP71E, RhoGAP92B, RhoGAP93B, RhoGEF2, RhoL, rl, robl, Rtnl1, SCAR, Sep2, shi, spir, sprt, sqh, Ssrp, stai, svr, Synd, tacc, Tes, trio, tsr, tsu, Ubc10, unc-104, Vap33, Vav, vib, Vps16A, Vrp1, wac, wash, WASp

\section{Golgi-related}

CG31145, Syx17, sll, pns, BicD, PAPLA1, Syx4, CG9773, IPIP, Trs33, Snap29, cni, Bet1, CG3662, CG5447, Bet5, Vti1b, Gga, Vps29, Arf51F, CG30423, LPCAT, CG11753, Rab8, CG9067, CG32069, Exo84, Trs31, Trs20, CG10344, Cam, Arl2, Rab39, CG15168, CG43322, Lerp, Clc, Gos28, Dab, comm, brn, Tomosyn, CG5934, zetaCOP, Arf102F, CG33116, Fer1HCH, Rab2, Trs23, CG7536, CG16865, ATP6AP2, Ykt6, vib, Nhe1, Sec10, Syx16, Doa, ksh, CG15099, Fer2LCH, Snx1, Zip99C, Rab11, Ccm3, CG5021, COX4, Efr, pod1, Rab1, Sec6, park, Bet3, CG5382, boca, Tango5, Rint1, prd1, Snx3, Rab19, CG5196, Fur2, Use1, strat, CG14511, wash, CG33635, Tango14, Golgin104, beta4GalNAcTB, Sec15, CG1116, c11.1, Sec5, FucTB, CG32485, Syx5, CG8314, RhoGAP1A, Snap24, ema, CG5510, IdlCp, PHGPx, CG14232, Arl5, asrij, Yip1d1, Arfip, Membrin, Madm, CG4645, Arl1, Chc, Rab18, Rich, GPHR, alphaSnap, epsilonCOP, Arf79F

Exocytosis

Arf102F, Arf51F, Arf79F, Arl1, Arl2, Atet, BicD, car, CCDC53, CG31935, Chc, comm, Dab, disp, Esyt2, Exo70, Exo84, fwe, pck, Rab11, Rab35, Rab7, Rab8, Rap1, Rop, Rrp47, Sec10, Sec15, Sec5, Sec6, shi, shrb, Snap24, Snap29, stac, stmA, Syngr, Syt1, Syx17, Syx1A, Syx5, Thor, Tomosyn, TSG101, Vamp7, Vap33, Vps11, Vps20, Vps24, Vps60, WASp

Adhesion

Fas3, sd, CASK, rhea, NijA, Arpc4, Sema2b, Ggamma1, CCDC53, CG34325, NijB, RhoL, Itgbn, parvin, brn, alpha-Cat, Sap-r, cold, tx, eff, CG17278, Bsg, Arpc1, pyr, Flo2, cindr, Hem, Rap1, FER, wash, trio, shi, Flo1, Psn, muskelin, egh, SCAR

Autophagy

Arl8, Atg12, Atg3, Atg4a, Atg6, Atg7, Atg8a, Atg9, bchs, Bl-1, Blos1, Blos2, BRWD3, Buffy, Cam, car, Ccz1, CG11781, CG11975, CG12163, CG32039, CG42554, CG5445, CG5676, CG6878, CG8155, CG8270, Chc, CHMP2B, crq, CycC, Doa, DOR, Dronc, ema, fab1, Fis1, Fkbp39, Iml1, Lerp, It, MED24, Nprl3, Nup44A, park, Pi3K59F, Rab1, Rab11, Rab19, Rab2, Rab21, RagA-B, RagC-D, Ras85D, Rheb, rl, rpr, Sap-r, Sec61gamma, SH3PX1, shi, shrb, Snap29, SNF4Agamma, Sod1, Syx13, Syx17, Tango5, Trpml, Ubc6, Utx, Uvrag, Vamp7, Vps11, Vps15, Vps16A, Vps25, Vps28, Vps36, Vps39, zda

D. melanogaster orthologs are listed and their predicted function is based on www.flybase.org.

Comparative Morphology and

Transcriptome of MGHs
J Innate Immun 2022;14:335-354

DOI: $10.1159 / 000520110$ 


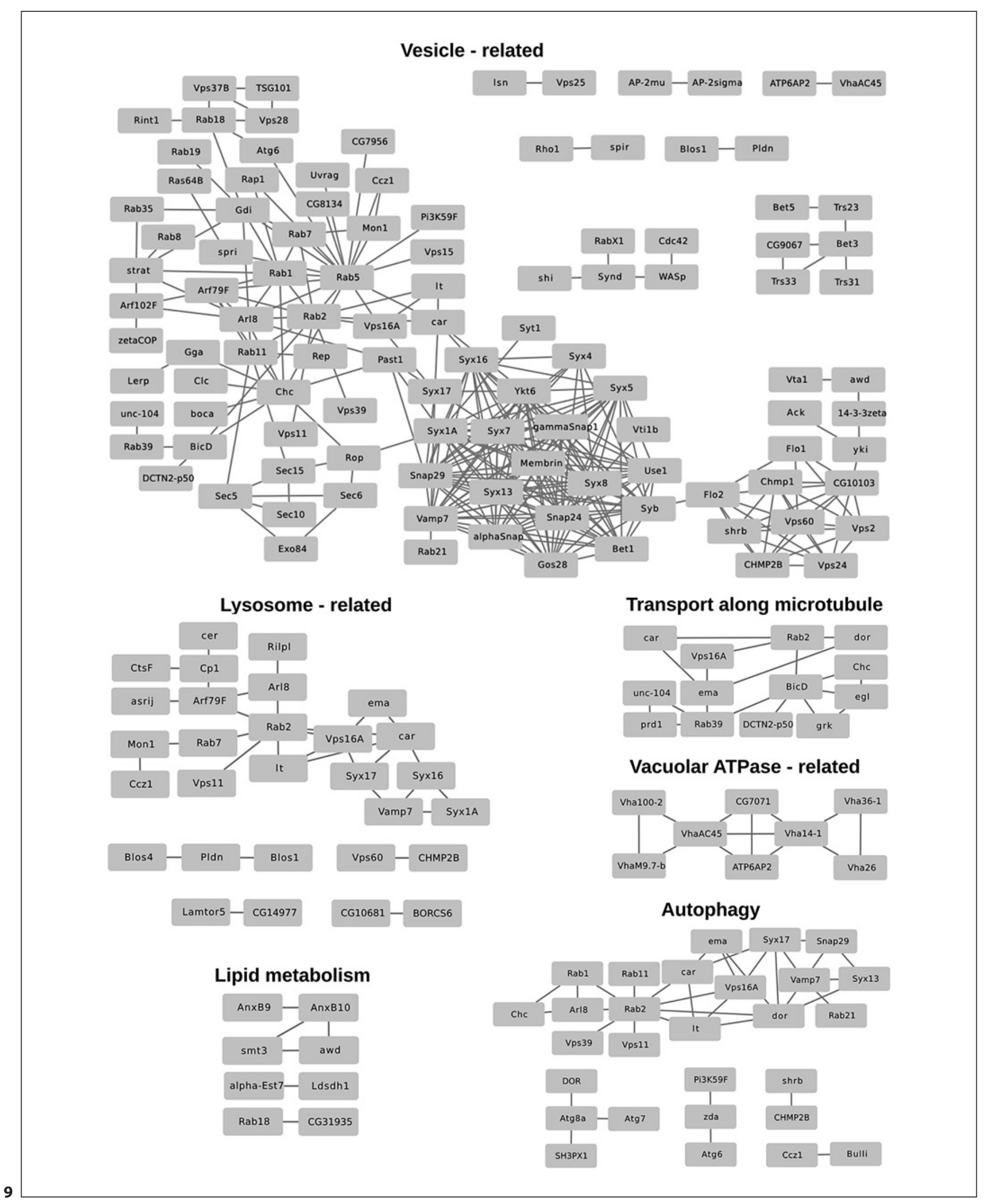

(For legend see next page.) 
highly expressed in both cell types. In addition, the ortho$\log$ of another lamellocyte-specific marker, atilla [41, 42], was highly expressed by MGHs (online suppl. Table S1). To sum, these molecules are highly expressed in at least 2 encapsulating cell types and feasibly involved in MGHmediated encapsulation of parasites.

In addition to analyzing similarities between D. melanogaster lamellocytes and $D$. ananassae $\mathrm{MGH}$, we also looked for molecules that were expressed in MGHs but did not have $D$. melanogaster orthologs because they may reveal MGH-specific features. In this domain, specifically interesting were a family of 12 genes (FBgn0096551, FBgn0096550, FBgn0096554, FBgn0096359, FBgn0097309, FBgn0099661, FBgn0096553, FBgn0096357, FBgn0096354, FBgn0096360, FBgn0096555, FBgn0096355), which represent predicted members of the Hemolysin E (also named as Cytolysin A) family. Members of this family are pore-forming toxins encoded by several bacterial species [43] and therefore feasibly contribute to the effective immune response against parasites.

\section{Discussion}

MGHs mediate a highly efficient immune response against parasitoid wasps $[12,23,24]$. To reveal the structural and functional features of MGHs, in this study we carried out electron microscopic and next-generation transcriptomic analyses of $D$. ananassae MGHs, activated plasmatocytes, and uninduced blood cells for comparison. These revealed a vesicle-rich intracellular organization, existence of a multiform dense body system, and unique transcriptomic characteristics in MGHs. The ultrastructural and transcriptomic findings were consistent with one another, and together provided mechanistic insights into the extremely fast cellular growth and high motility shown by MGHs, and their efficient defense against parasitoids.

\section{Vesicle-Rich Intracellular Organization of MGHs}

MGHs are generated upon parasitoid wasp infection. Their precursors, the spherical plasmatocytes, elongate and lose their phagocytic and dividing capacity in re-

Fig. 9. Predicted physical interaction networks of vesicle-, vacuole-, lysosome-, autophagy-, and lipid metabolism-related proteins encoded by genes upregulated in the MGHs based on interactions of $D$. melanogaster orthologs (www.flybase.org). The $D$. ananassae equivalents of the respective candidates are listed in online suppl. Tables S1 and S2.

Comparative Morphology and

Transcriptome of MGHs sponse to infection. Subsequently, these elongated cells fuse with each other and form the terminally differentiated MGHs, which have the capacity to encapsulate invaders [12]. Previously, in $Z$. indianus, we found that MGHs carry relatively few vesicles and possess a spongelike overall ultrastructure formed by an irregular cytoplasmic canalicular system [13]. By contrast, our current analysis of $D$. ananassae MGHs revealed a large number of diverse vesicular systems and a canalicular organization which is mostly localized at the cell periphery. In electron microscopic images, the organization of vesicular systems was consistent with roles in storage, transport, and remodeling of intracellular materials. In addition, vesicles appeared to contribute to rapid formation of an elaborate membrane system, particularly noticeable at the cell periphery. Further, light vesicles developed into an extended reticular system occupying large areas of MGHs. Using immunolabeling, we demonstrated that the 7C5 protein, specifically localized in MGH plasma membranes, also is apparent in the post-Golgi area where vesicular, tubular, and cisternal organelles appeared to be en route to or already fused with the plasma membrane providing rapid growth of the cell envelope (Fig. 7c, d, e). It is possible that in addition to increasing cell size by direct synthesis of new membrane areas, these fusion events secreted transported content by exocytosis. Finally, we detected a large number of lipid droplets in MGHs (Fig. 2; online suppl. Fig. S3), whose appearance was consistent with upregulated lipid storage-related genes (Table 1; online suppl. Tables S1 and S2; Fig. 9), suggesting that lipids may serve as a reservoir for cholesterol and acyl-glycerols, which are required for the formation and maintenance of the outstretched membrane system [44].

\section{Multiform Dense Body System}

Similar to our previous observations in Z. indianus [13], we found an MDBS in D. ananassae MGHs, which appeared to contribute to the formation of an electron-dense layer along the attachment site with the parasitoid. The well-organized, lamellar structure of this electron-dense layer likely facilitates strong and stable attachment of MGHs to parasitoids, encapsulation, and possibly degradation. While its composition remains elusive, our data demonstrate that assembly of the electron-dense layer is facilitated by a direct transfer route of materials from the Golgi apparatus, suggesting a dedicated cellular mechanism behind its development. Providing further insights, we detected acidic vesicles and an acidic layer, whose location corresponded with that of the electron-dense layer (Fig. 4). Although due to technical limitations we could not unambiguously colo-

Innate Immun 2022;14:335-354 349 


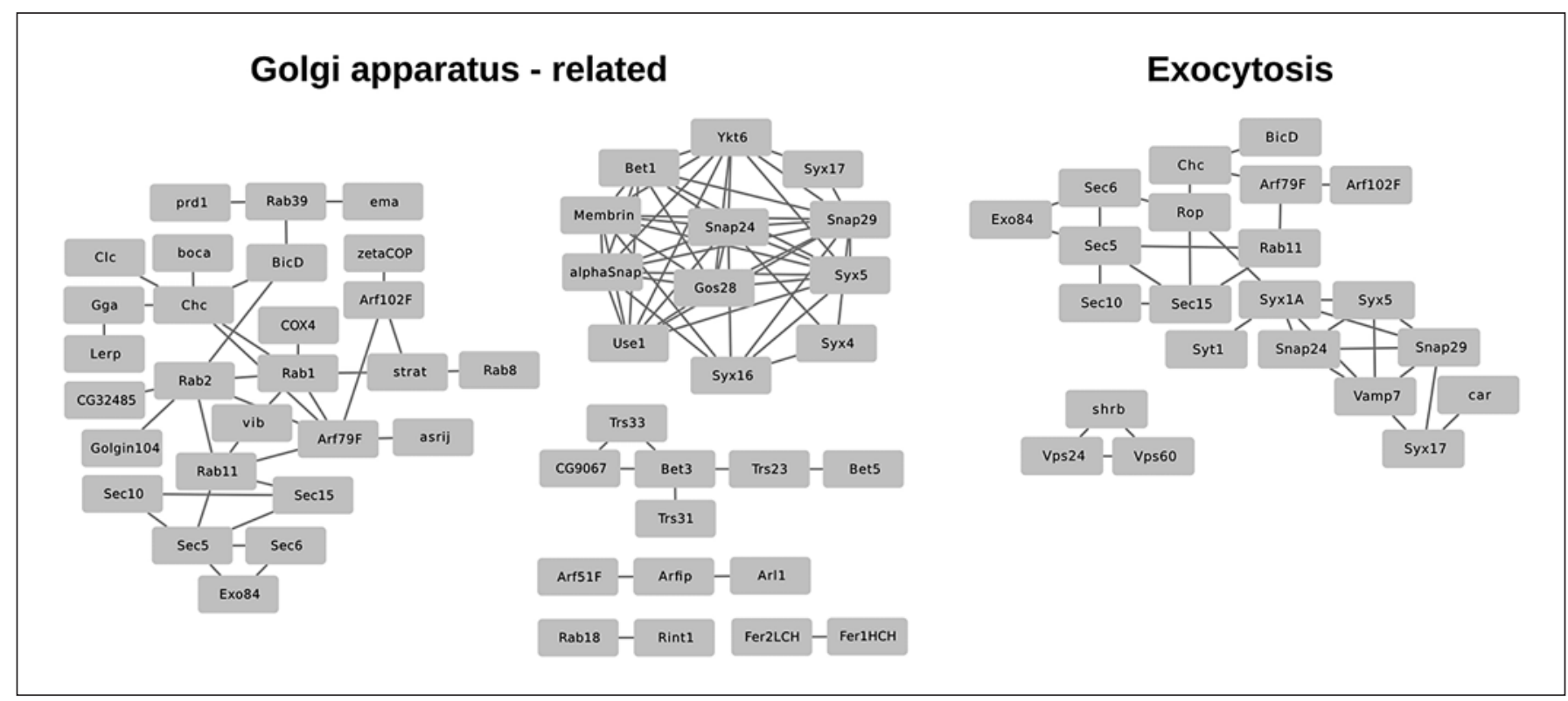

Fig. 10. Predicted physical interaction networks of proteins encoded by genes upregulated in the MGHs involved in Golgi apparatus-related processes and exocytosis. The D. melanogaster orthologs are shown. Networks are based on the available information on www.flybase.org.

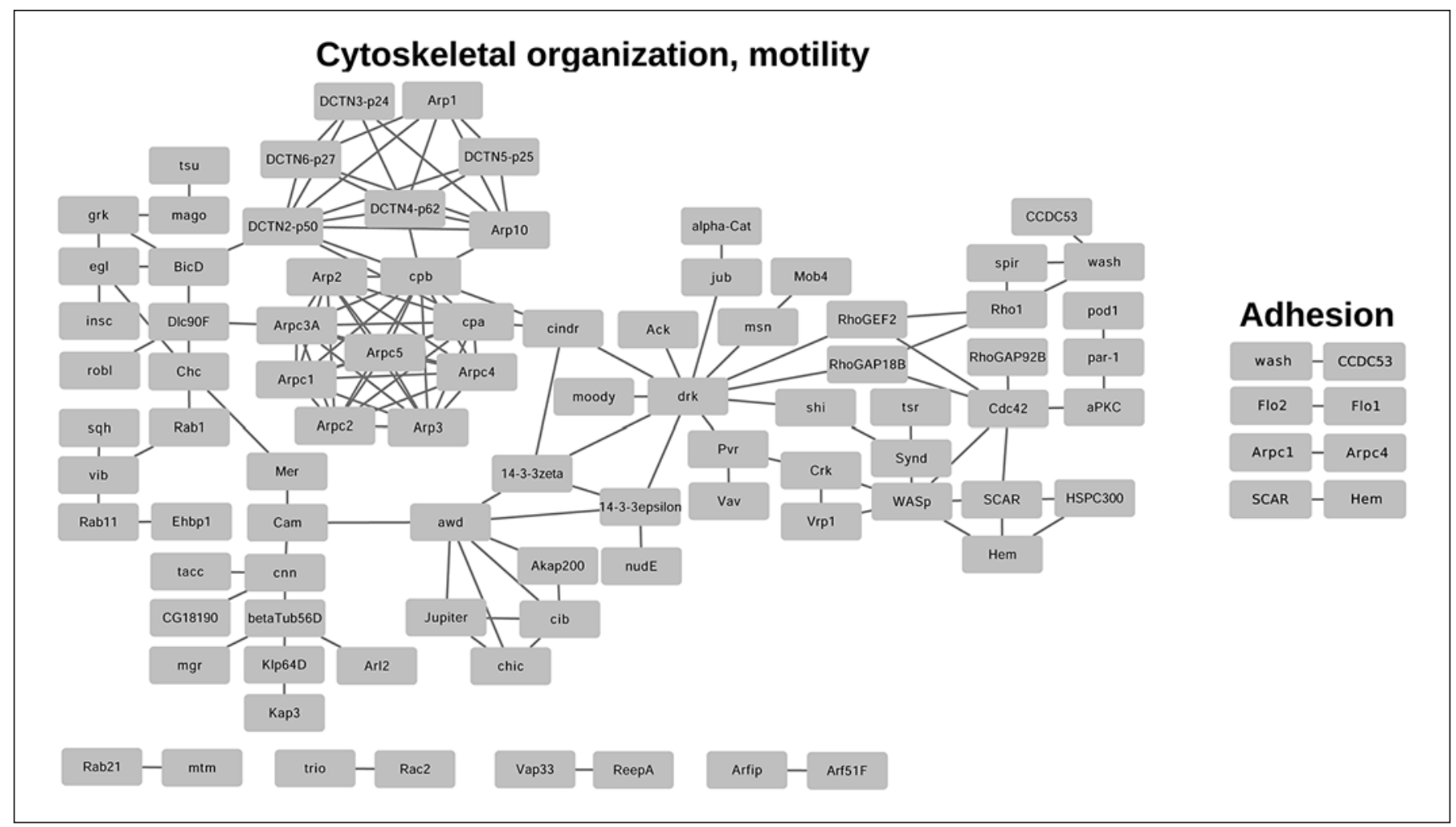

Fig. 11. Predicted physical interaction networks of proteins encoded by genes upregulated in the MGHs involved in cytoskeletal organization, motility, and adhesion. The D. melanogaster orthologs are shown. Networks are based on the available information on www.flybase.org. 
calize these in 1 conclusive experiment, we presume that the electron-dense material and the acidic structures were identical or at least very closely related.

An important question regarding the MGH-mediated encapsulation is whether melanization is required for a successful immune response. In D. melanogaster, melanin is generated by blood cells and participates in the melanization of the capsule [10], but PPO3, which is required for this process, does not have an ortholog in D. ananassae [11]. Our transcriptional analysis furthermore revealed that neither PPO1 nor PPO2, which could also facilitate melanization, was expressed by MGHs. In addition, neither of the orthologs of MP1, Sp7, and Hayan, which activate the melanization cascade $[45,46]$, was expressed by MGHs, confirming that the encapsulation reaction mediated by MGHs does not require melanization.

In contrast to melanization, a large number of genes involved in autophagy were highly expressed by MGHs (Table 1; online suppl. Tables S1 and S2; Fig. 9). In addition, and in consistent with this, we detected a large number of autophagosomes in MDBS areas (online suppl. Fig. S3), where lysosomal self-degradation is the most likely to take place. Further, gene expression analysis revealed high expression of multiple genes that encode vacuolartype ATPase subunits (Fig. 9), which could contribute to intensified proton-pumping activity and thus acidification. Since we already noted a correspondence between the electron-dense areas and acidic structures, it is plausible that acidification in these areas is rendered by vacuolar-type ATPases. However, using LysoTracker, we also noted that retention of the dye was much lower in $\mathrm{MGH}$ vesicles than in activated plasmatocytes (online suppl. Fig. S2b). This may be explained by our finding that the D. ananassae gene FBgn0093624, an ortholog of the human ABCC4 gene, was significantly enriched in MGHs compared to activated plasmatocytes. This gene encodes a P-glycoprotein, which has been shown to recognize and transport aromatic and hydrophobic compounds, such as the LysoTracker dye, by efflux pumping [47]. Therefore, it is possible that the extremely fast decrease of the LysoTracker fluorescence intensity in acidic organelles of the MGHs was rendered by P-glycoprotein activity.

Transcriptomic Insights into Recognition,

Encapsulation, and Killing of Parasitoids

In addition to the ultrastructural observations, our transcriptomic analyses provided insights into the immune response mediated by MGHs. As characteristic steps in the process, we concentrated on the recognition, encapsulation, and killing of parasites.

Comparative Morphology and

Transcriptome of MGHs
With regard to the recognition, significant enrichment of 2 D. ananassae-specific genes was particularly noticeable in MGHs. These were FBgn0099042 and FBgn0089610, which encode proteins containing fibrinogen $\mathrm{C} 2$ domains and $\mathrm{N}$-terminal signal peptides. This finding could be significant because in invertebrates fibrinogen $\mathrm{C} 2$ domain-containing proteins have been associated with recognition and precipitation of parasitederived molecules [48]. Further, orthologs of the D. melanogaster genes croquemort and lozenge were highly expressed by MGHs (online suppl. Tables S1 and S2). Croquemort promotes apoptotic cell clearance in D. melanogaster plasmatocytes [49], whereas lozenge, a marker for D. melanogaster crystal cells, is known to be involved in transcriptional regulation [50]. In addition, orthologs of genes involved in JNK signaling, such as bendless, Pvr, Alg-2, CYLD, cpa, and Cdc42 [51-57], were highly expressed by MGHs. By contrast, elements of other signaling pathways relevant for immune response to pathogens in D. melanogaster (e.g., Toll, IMD, or JAK/STAT) were not enriched, underscoring that JNK signalization is likely a key mediator of MGH movement or stress response following infection.

Encapsulation is a general reaction against foreign particles and parasitoids. However, the evolutionary origin of the encapsulating blood cells in different species of Drosophilidae remains poorly understood. While here we identified several genes that are highly expressed in encapsulating D. ananassae MGHs and D. melanogaster lamellocytes (online suppl. Table S5), the elimination of the parasitoids is more effective in $D$. ananassae $[12,23]$. This may be due to major differences in the killing mechanisms between the 2 species, the notion of which is somewhat supported, but not entirely explained, by the lack of melanization by $D$. ananassae [12]. The 12 predicted members of Hemolysin family, encoded in the genome of $D$. ananassae but not $D$. melanogaster, may provide further insights into the differences of parasite elimination. Hemolysins are pore-forming toxins that can lyse erythrocytes and mammalian cells and are encoded by several bacterial species [43]. In addition to D. ananassae, these genes are present in the genomes of several other species of the ananassae subgroup of Drosophilidae (as D. malerkotliana, D. bipectinata, D. parabipectinata, D. pseudoananassae, D. varians, and D. ercepeace). Lateral transfer of toxin genes from prokaryotic organisms or phages to eukaryotes, such as the cytolethal distending toxin B [58] or parasitoid-killing factors [59], has previously been described. Therefore, it is possible that horizontal gene transfer may have been a key mechanism for

J Innate Immun 2022;14:335-354 351 
acquisition of new phenotypes in species of the ananassae subgroup, which broadened the repertoire, complexity, and effectiveness of their immune response and defense. While the precise recognition, encapsulation, and killing mechanisms by MGHs remain elusive until further experimental evidences, these transcriptomically identified molecules already provide insights and illuminate a path toward genetic dissection of such mechanisms.

\section{Giant Cell Exosomes}

Our electron microscopic analyses have revealed another noticeable feature of $D$. ananassae MGHs, namely, the intensive budding of plasma membrane into the hemolymph (Fig. 6, 7d, e). Membrane-wrapped vesicles had a diameter of $50-80 \mathrm{~nm}$, and thus we named them as GCEs [60]. Consistent with this observation, we found high expression of the following D. melanogaster orthologs in MGHs: (i) tetraspanins CD81, CD82, CD37, and CD63, known to be enriched in exosomes [61]; (ii) Rab35, which facilitate exosome biogenesis [62]; and (iii) several genes related to endosomal sorting complex required for transport, which mediates exosome biogenesis [60]. It is known that cells from bacteria to vertebrates produce diverse exosomes, which cargo proteins, lipids, and nucleic acids either in their internal compartment or displayed on the exosome surface $[63,64]$. Cargo molecules may mediate signaling between cells and, in some cases, are associated with diseases, such as cancer and infections [60,65-68]. There are limited data concerning the extracellular vesicles in insect species. In Drosophila, it was shown that hemocytes can take up dsRNA from infected cells, produce virus-derived complementary DNAs, and de novo synthesize secondary viral siRNAs. These viral siRNAs are secreted in exosomelike vesicles for protection against virus challenge [69]. Moreover, released exosomes may shape mating-specific behaviors in other individuals [70] or mediate targeted long-range signaling, for example, by Hedgehog proteins [71]. In MGHs, it is feasible that GCEs mediate signaling to other tissues to maintain homeostasis after parasitoid infection, or directly contribute to the killing of parasitoids. The considerable number of exosome related genes expressed in MGHs could facilitate future research that elucidates the specific physiological relevance of GCEs.

\section{Conclusion}

Our study provided new insights into an extremely effective innate immune response mediated by MGHs. Both the unique ultrastructure and gene expression pro- file of $D$. ananassae MGHs highlight a dedicated defense mechanism against parasitoids. As a model, the capsule formation by MGHs could serve to better understand similar defense mechanisms in vertebrates, such as the foreign body-type granulomas, whereas genomic characterization of hemocyte subpopulations could facilitate target discovery for diagnostics and molecule-targeted therapies.

\section{Acknowledgments}

We are grateful to Olga Kovalcsik, Henrietta Kovács, Anita Balázs, and Sarolta Pálfia for the technical help. This research was supported by grants from the Hungarian National Science Foundation, NKFI K128762 (G.C.), NKFI K135877 (I.A.), NKFIH KKP129797 (G.J.), the Swedish Research Council, project number: 2018-05114 (D.H.), the BO/00552/20/8 János Bolyai Research Scholarship of the Hungarian Academy of Sciences (G.C.), the GINOP-2.3.2-15-2016-00035 (E.K) and the Doctoral School of Biology, University of Szeged (L.B.M. and Z.L.).

\section{Statement of Ethics}

Animal experiments that conform to internationally accepted standards have been approved by the appropriate institutional review body (CS/I01/765/2018).

\section{Conflict of Interest Statement}

The authors have no conflicts of interest to declare.

\section{Funding Sources}

Costs of the RNA isolation from uninduced blood cells, charges of the work with the Olympus FV1000 confocal LSM microscope, costs of laboratory equipment required for the work, and the publication fee are supported by the Grant NKFI K128762 (G.C.). Tissue culture media and chemicals for hybridoma culture, antibodies, costs of insect stocks and maintenance, costs of chemicals required for electron microscopic analysis, costs of cDNA library preparation and next-generation RNA sequencing, and salary are supported by the Grant NKFI K135877 (I.A.). Costs for the electron microscopic analysis and regents for LysoTracker staining are supported by the Grant NKFIH KKP129797 (G.J.). This study was also supported by Swedish Research Council, project number: 2018-05114 (D.H.) and GINOP-2.3.2-15-2016-00035 (E.K.) for supply. Scholarships are funded by BO/00552/20/8 János Bolyai Research Scholarship of the Hungarian Academy of Sciences (G.C.) and Doctoral School of Biology, University of Szeged (L.B.M., Z.L.). 


\section{Author Contributions}

Gyöngyi Cinege processed the experimental design; prepared the sample for next-generation RNA sequencing; performed experiments with the LysoTracker dye; and prepared the manuscript. Lilla B. Magyar analyzed the comparative transcriptome data and gene clustering, performed the experiments with the LysoTracker dye, and contributed to the preparation of the manuscript. Attila L. Kovács prepared the sample and electron microscopic analysis of hemocytes and contributed to the preparation of the manuscript. Zita Lerner prepared the samples for electron microscopic analysis. Gábor Juhász processed the electron microscopic analysis of hemocytes. David Lukacsovich processed the RNA sequencing data and contributed to the bioinformatical analysis of RNA sequencing data and comparative transcriptome data. Jochen Winterer was involved in single-cell harvesting and cDNA library preparation for next-generation RNA sequencing. Tamás Lukacsovich was involved in cDNA library preparation and next-gener- ation RNA sequencing for uninduced samples, and in the discussion of the results. Zoltán Hegedüs contributed to the bioinformatical analysis of RNA sequencing data and the comparative transcriptome data; and gene clustering. Éva Kurucz produced the monoclonal antibodies. Dan Hultmark analyzed the MGH-specific genes and discussed the results of the study. Csaba Földy was involved in experimental design, discussion of the results, and preparation of the manuscript. István Andó contributed to the experimental design, the production of monoclonal antibodies, the discussion of the results, and the preparation of the manuscript.

\section{Data Availability Statement}

The data that support the finding of this study are openly available in the NCBI GEO repository at: https://www.ncbi.nlm.nih. gov/geo/query/acc.cgi?acc=GSE180960, reference number GSE180960, reviewer token: krodkaeervmpruz.

\section{References}

1 Evans CJ, Hartenstein V, Banerjee U. Thicker than blood: conserved mechanisms in Drosophila and vertebrate hematopoiesis. Dev Cell. 2003;5:673-90.

2 Hoffmann JA. The immune response of Drosophila. Nature. 2003;426(6962):33-8.

3 Gilmore TD, Wolenski FS. NF- $\kappa B$ : where did it come from and why? Immunol Rev. 2012; 246(1):14-35.

4 Rizki TM, Rizki RM. Properties of the larval hemocytes of Drosophila melanogaster. Experientia. 1980;36(10):1223-6.

5 Lanot R, Zachary D, Holder F, Meister M. Postembryonic hematopoiesis in Drosophila. Dev Biol. 2001;230:243-57.

6 Márkus R, Laurinyecz B, Kurucz E, Honti V, Bajusz I, Sipos B, et al. Sessile hemocytes as a hematopoietic compartment in Drosophila melanogaster. Proc Natl Acad Sci USA. 2009; 106:4805-9.

7 Honti V, Csordás G, Kurucz É, Márkus R, Andó I. The cell-mediated immunity of Drosophila melanogaster: hemocyte lineages, immune compartments, microanatomy and regulation. Dev Comp Immunol. 2014;42(1): 47-56.

8 Kim-Jo C, Gatti JL, Poirié M. Drosophila cellular immunity against parasitoid wasps: a complex and time-dependent process. Front Physiol. 2019;10:603.

9 Brehélin M. Comparative study of structure and function of blood cells from two Drosophila species. Cell Tissue Res. 1982;221: 607-15.

10 Nappi A, Poirié M, Carton Y. The role of melanization and cytotoxic by-products in the cellular immune responses of Drosophila against parasitic wasps. Adv Parasitol. 2009; 70:99-121.

11 Dudzic JP, Kondo S, Ueda R, Bergman CM, Lemaitre B. Drosophila innate immunity: regional and functional specialization of prophenoloxidases. BMC Biol. 2015;13:81.
12 Márkus R, Lerner Z, Honti V, Csordás G, Zsámboki J, Cinege G, et al. Multinucleated giant hemocytes are effector cells in cell-mediated immune responses of Drosophila. J Innate Immun. 2015;7:340-53.

13 Cinege G, Lerner Z, Magyar LB, Soós B, Tóth $\mathrm{R}$, Kristó I, et al. Cellular immune response involving multinucleated giant hemocytes with two-step genome amplification in the drosophilid Zaprionus indianus. J Innate Immun. 2020;12:257-72.

14 Miron RJ, Bosshardt DD. Multinucleated giant cells: good guys or bad guys? Tissue Eng Part B Rev. 2018;24:53. 24

15 Pagán AJ, Ramakrishnan L. The formation and function of granulomas. Annu Rev Immunol. 2018;36(1):639-65.

16 Brodbeck WG, Anderson JM. Giant cell formation and function. Curr Opin Hematol. 2009;16(1):53-7.

17 Sakai H, Okafuji I, Nishikomori R, Abe J, Izawa $\mathrm{K}$, Kambe N, et al. The CD40-CD40L axis and IFN- $\gamma$ play critical roles in Langhans giant cell formation. Int Immunol. 2012;24:515.

18 Martínez-Girón R, Pantanowitz L. Lower respiratory tract viral infections: Diagnostic role of exfoliative cytology. Diagn Cytopathol. 2017;45(7):614-20.

19 Daly JL, Simonetti B, Klein K, Chen KE, Williamson MK, Antón-Plágaro C, et al. Neuropilin-1 is a host factor for SARS-CoV-2 infection. Science. 2020;370(370):861-5.

20 Anderson RS. Polykaryon formation by mercenaria hemocytes. Biol Bull. 1987;172:2.

21 Favery B, Quentin M, Jaubert-Possamai S, Abad P. Gall-forming root-knot nematodes hijack key plant cellular functions to induce multinucleate and hypertrophied feeding cells. J Insect Physiol. 2016;84:60-9.

22 Galko MJ, Krasnow MA. Cellular and genetic analysis of wound healing in Drosophila larvae. PLoS Biol. 2004;2(8):e239.
23 Kacsoh BZ, Schlenke TA. High hemocyte load is associated with increased resistance against parasitoids in Drosophila suzukii, a relative of D. melanogaster. PLoS One. 2012;7:e34721.

24 Kacsoh BZ, Bozler J, Schlenke TA. A role for nematocytes in the cellular immune response of the Drosophilid Zaprionus indianus. Parasitology. 2014;141:697-715.

25 Földy C, Darmanis S, Aoto J, Malenka RC, Quake SR, Südhof TC. Single-cell RNAseq reveals cell adhesion molecule profiles in electrophysiologically defined neurons. Proc Nat Acad Sci USA. 2016;113:E5222-31.

26 Lukacsovich D, Winterer J, Que L, Luo W, Lukacsovich T, Földy C. Single-cell RNA-seq reveals developmental origins and ontogenetic stability of neurexin alternative splicing profiles. Cell Rep. 2019;27:3752-e4.

27 Yang J, Shen HB. MemBrain-contact 2.0: a new 2-stage machine learning model for the prediction enhancement of transmembrane protein residue contacts in the full chain. Bioinformatics. 2018;34(2):230-8.

28 Boyle EI, Weng S, Gollub J, Jin H, Botstein D, Cherry JM, et al. GO: TermFinder-open source software for accessing gene ontology information and finding significantly enriched gene ontology terms associated with a list of genes. Bioinformatics. 2004;20:3710-5.

29 Supek F, Bošnjak M, Škunca N, Šmuc T. REVIGO summarizes and visualizes long lists of gene ontology terms. PLoS One. 2011;6: e21800.

30 Shannon P, Markiel A, Ozier O, Baliga NS, Wang JT, Ramage D, et al. Cytoscape: a software environment for integrated models of biomolecular interaction networks. Genome Res. 2003;13:2498-504.

31 Lüllmann-Rauch R. History and morphology of the lysosome. Lysosomes. Medical intelligence unit. Boston, MA: Springer; 2005. p. $1-16$.
Comparative Morphology and

Transcriptome of MGHs
J Innate Immun 2022;14:335-354

DOI: $10.1159 / 000520110$ 
32 Eskelinen EL, Reggiori F, Baba M, Kovács AL, Seglen PO. Seeing is believing: the impact of electron microscopy on autophagy research. Autophagy. 2011;7(9):935-56.

33 Appelqvist $\mathrm{H}$, Wäster $\mathrm{P}$, Kågedal $\mathrm{K}$, Öllinger $\mathrm{K}$. The lysosome: from waste bag to potential therapeutic target. J Mol Cell Biol. 2013;5(4): $214-26$.

34 Littleton JT. A genomic analysis of membrane trafficking and neurotransmitter release in Drosophila. J Cell Biol. 2000;150(2):F77-82.

35 Cattenoz PB, Sakr R, Pavlidaki A, Delaporte C, Riba A, Molina N, et al. Temporal specificity and heterogeneity of Drosophila immune cells. EMBO J. 2020;39(12):e104486.

36 Cho B, Yoon SH, Lee D, Koranteng F, Tattikota SG, Cha N, et al. Single-cell transcriptome maps of myeloid blood cell lineages in Drosophila. Nat Commun. 2020 Sep 8;11(1): 4483.

37 Fu Y, Huang X, Zhang P, van de Leemput J, Han Z. Single-cell RNA sequencing identifies novel cell types in Drosophila blood. J Genet Genomics. 2020;47(4):175-86.

38 Leitao AB, Arunkumar R, Day JP, Geldman EM, Morin-Poulard I, Crozatier M, et al. Constitutive activation of cellular immunity underlies the evolution of resistance to infection in Drosophila. eLife. 2020;9:e59095.

39 Tattikota SG, Cho B, Liu Y, Hu Y, Barrera V, Steinbaugh MJ, et al. A single-cell survey of drosophila blood. eLife. 2020;9:e54818.

40 Wan B, Belghazi M, Lemauf S, Poirié M, Gatti JL. Proteomics of purified lamellocytes from Drosophila melanogaster HopTum-1 identifies new membrane proteins and networks involved in their functrions. Insect Biochem Mol Biol. 2021;134:103584.

41 Kurucz E, Váczi B, Márkus R, Laurinyecz B, Vilmos P, Zsámboki J, et al. Definition of drosophila hemocyte subsets by cell-type specific antigens. Acta Biol Hung. 2007;58:95-111.

42 Honti V, Kurucz E, Csordás G, Laurinyecz B, Márkus R, Andó I. In vivo detection of lamellocytes in Drosophila melanogaster. Immunol Lett. 2009;126:83-4.

43 Wallace AJ, Stillman TJ, Atkins A, Jamieson SJ, Bullough PA, Green, et al. E. coli Hemolysin E (HlyE, ClyA, SheA): X-ray crystal structure of the toxin and observation of membrane pores by electron microscopy. Cell. 2000;100:265-76.

44 Lange Y, Tabei SM, Ye J, Steck TL. Stability and stoichiometry of bilayer phospholipidcholesterol complexes: relationship to cellular sterol distribution and homeostasis. Biochemistry. 2013;52:6950-9.

45 Tang H, Kambris Z, Lemaitre B, Hashimoto C. Two proteases defining a melanization cascade in the immune system of Drosophila. J Biol Chem. 2006;281(38):28097-104.
46 Dudzic JP, Hanson MA, Iatsenko I, Kondo S, Lemaitre B. More than black or white: melanization and toll share regulatory serine proteases in Drosophila. Cell Rep. 2019;27(4): 1050-61.e3.

47 Zhitomirsky B, Farber H, Assaraf YG. LysoTracker and mitotracker red are transport substrates of P-glycoprotein: implications for anticancer drug design evading multidrug resistance. J Cell Mol Med. 2018;22:2131-41.

48 Adema CM, Hertel LA, Miller RD, Loker ES. A family of fibrinogen-related proteins that precipitates parasite-derived molecules is produced by an invertebrate after infection. Proc Natl Acad Sci USA. 1997;94:8691-6.

49 Franc NC, Dimarcq JL, Lagueux M, Hoffmann J, Ezekowitz RA. Croquemort, a novel Drosophila hemocyte/macrophage receptor that recognizes apoptotic cells. Immunity. 1996;4(5):431-43.

50 Wildonger J, Sosinsky A, Honig B, Mann RS. Lozenge directly activates argos and klumpfuss to regulate programmed cell death. Genes Dev. 2005;19:1034-9.

51 La Marca JE, Richardson HE. Two-faced: roles of JNK signalling during tumourigenesis in the Drosophila model. Front Cell Dev Biol. 2020;8:42.

52 Ma X, Shao Y, Zheng H, Li M, Li W, Xue L. Src42A modulates tumor invasion and cell death via Ben/dUevla-mediated JNK activation in Drosophila. Cell Death Dis. 2013; 4(10):e864.

53 Ishimaru S, Ueda R, Hinohara Y, Ohtani M, Hanafusa H. PVR plays a critical role via JNK activation in thorax closure during Drosophila metamorphosis. EMBO J. 2004;23(20): 3984-94.

54 Tsuda M, Seong KH, Aigaki T. POSH, a scaffold protein for JNK signaling, binds to ALG2 and ALIX in Drosophila. FEBS Lett. 2006; 580(13):3296-300.

55 Xue L, Igaki T, Kuranaga E, Kanda H, Miura $\mathrm{M}, \mathrm{Xu}$ T. Tumor suppressor CYLD regulates JNK-induced cell death in Drosophila. Dev Cell. 2007;13(3):446-54.

56 Jezowska $B$, Fernández BG, Amândio $A R$, Duarte P, Mendes C, Brás-Pereira C, et al. A dual function of Drosophila capping protein on DE-cadherin maintains epithelial integrity and prevents JNK-mediated apoptosis. Dev Biol. 2011;360(1):143-59.

57 Genova JL, Jong S, Camp JT, Fehon RG. Functional analysis of Cdc42 in actin filament assembly, epithelial morphogenesis, and cell signaling during Drosophila development. Dev Biol. 2000;221(1):181-94.
58 Verster KI, Wisecaver JH, Karageorgi M, Duncan RP, Gloss AD, Armstrong EE, et al. Horizontal transfer of bacterial cytolethal distending toxin $\mathrm{B}$ genes to insects. Mol Biol Evol. 2019;36(10):2105-10.

59 Gasmi L, Sieminska E, Okuno S, Ohta R, Coutu C, Vatanparast M, et al. Horizontally transmitted parasitoid killing factor shapes insect defense to parasitoids. Science. 2021; 373(6554):535-41.

60 Pegtel DM, Gould SJ. Exosomes. Annu Rev Biochem. 2019;88(1):487-514.

61 Escola JM, Kleijmeer MJ, Stoorvogel W, Griffith JM, Yoshie O, Geuze HJ. Selective enrichment of tetraspan proteins on the internal vesicles of multivesicular endosomes and on exosomes secreted by human B-lymphocytes. J Biol Chem. 1998;273:20121-7.

62 Hsu C, Morohashi Y, Yoshimura S, Manrique-Hoyos N, Jung S, Lauterbach MA, et al. Regulation of exosome secretion by Rab35 and its GTPase-activating proteins TBC1D10A-C. J Cell Biol. 2010;189:223-32.

63 Colombo M, Raposo G, Théry C. Biogenesis, secretion, and intercellular interactions of exosomes and other extracellular vesicles. Annu Rev Cell Dev Biol. 2014;30:255-89.

64 Cocucci E, Meldolesi J. Ectosomes and exosomes: shedding the confusion between extracellular vesicles. Trends Cell Biol. 2015;25: 364-72.

65 Robbins PD, Morelli AE. Regulation of immune responses by extracellular vesicles. Nat Rev Immunol. 2014;14:195-208.

66 Desrochers LM, Antonyak MA, Cerione RA. Extracellular vesicles: satellites of information transfer in cancer and stem cell biology. Dev Cell. 2016;37:301.

67 Iraci N, Leonardi T, Gessler F, Vega B, Pluchino $S$. Focus on extracellular vesicles: physiological role and signalling properties of extracellular membrane vesicles. Int J Mol Sci. 2016;17(2):171

68 Altan-Bonnet N. Extracellular vesicles are the Trojan horses of viral infection. Curr Opin Microbiol. 2016;32:77-81.

69 Tassetto M, Kunitomi M, Andino R. Circulating immune cells mediate a systemic RNAibased adaptive antiviral response in Drosophila. Cell. 2017;169(2):314-25.e13.

70 Beer KB, Wehman AM. Mechanisms and functions of extracellular vesicle release in vivo-What we can learn from flies and worms. Cell Adh Migr. 2017;11(2):135-50.

71 Matusek T, Wendler F, Polès $S$, Pizette $S$, D'Angelo G, Fürthauer M, et al. The ESCRT machinery regulates the secretion and longrange activity of Hedgehog. Nature. 2014;516: 99-103. 